TRANSACTIONS OF THE

AMERICAN MATHEMATICAL SOCIETY

Volume 360, Number 12, December 2008, Pages 6711-6742

S 0002-9947(08)04538-8

Article electronically published on July 24, 2008

\title{
STRONG VARIATIONAL AND JUMP INEQUALITIES IN HARMONIC ANALYSIS
}

\author{
ROGER L. JONES, ANDREAS SEEGER, AND JAMES WRIGHT
}

\begin{abstract}
We prove variational and jump inequalities for a large class of linear operators arising in harmonic analysis.
\end{abstract}

\section{INTRODUCTION}

Variational and jump inequalities in probability, ergodic theory and harmonic analysis have been the subject of many recent articles (see [32, [5, 26, 27, .9, [10], and [28]). The purpose of this paper is to significantly extend and generalize some of the results for families of integral operators in harmonic analysis, and also to prove some previously open endpoint jump inequalities.

To fix notation we denote by $\mathfrak{I}$ a subset of $\mathbb{R}$ (or more generally an ordered index set). We consider real or complex valued functions $t \mapsto a_{t}$ defined on $\mathfrak{I}$ and define their $q$-variation to be

$$
\|a\|_{v_{q}}=\sup _{t_{1}<\cdots<t_{L}}\left(\sum_{\ell=1}^{L-1}\left|a_{t_{\ell+1}}-a_{t_{\ell}}\right|^{q}\right)^{1 / q}
$$

where the sup is taken over all finite increasing sequences $\left\{t_{1}<\cdots<t_{L}\right\}$ with $t_{i} \in \mathfrak{I}$.

Next we consider families of Lebesgue measurable functions $F=\left\{F_{t}: t \in \mathfrak{I}\right\}$ defined on $\mathbb{R}^{d}$ and define the strong q-variation of $F$ as the $v_{q}$ norm of $t \mapsto F_{t}(x)$; we denote it by $V_{q}(F)(x)$. If $\mathfrak{I}$ is assumed to be a countable index set, then $V_{q}(F)$ is a Lebesgue measurable function. However this countability assumption can be removed in many cases where the function $t \mapsto F_{t}(x)$ is continuous on $\mathfrak{I}$ for almost every $x$; this will always be the case in our applications. Here of course $\mathfrak{I}$ is endowed with the natural topology as a subset of $\mathbb{R}$.

Strong variational bounds follow from estimates of a more fundamental object, the $\lambda$-jump function $N_{\lambda}(F)$. One defines $N_{\lambda}(F)(x)$ to be the supremum of all integers $N$ for which there is an increasing sequence $0<s_{1}<t_{1} \leq s_{2}<t_{2} \leq \cdots \leq$ $s_{N}<t_{N}$ so that

$$
\left|F_{t_{\ell}}(x)-F_{s_{\ell}}(x)\right|>\lambda
$$

for each $\ell=1, \ldots, N$. It will sometimes be convenient to use a slight modification of the $\lambda$-jump function; namely, we define $\mathcal{N}_{\lambda}(F)(x)$ as the supremum of all nonnegative integers $N$ for which there exist $0<t_{1}<t_{2}<\cdots<t_{N}$ with the property

Received by the editors July 26, 2004 and, in revised form, April 23, 2007.

2000 Mathematics Subject Classification. Primary 42B15.

The second author was supported in part by NSF grant DMS 0200186.

(C)2008 American Mathematical Society 
that $\left|F_{t_{\ell+1}}(x)-F_{t_{\ell}}(x)\right|>\lambda, \ell=1, \ldots, N-1$. It is easy to see that

$$
\mathcal{N}_{\lambda}(F)(x) \leq N_{\lambda}(F)(x) \leq 2 \mathcal{N}_{\lambda / 2}(F)(x)
$$

and thus we can pass from one definition to the other without difficulty. The advantage of working with $N_{\lambda}$ is that it is 'effectively' subadditive; that is,

$$
N_{\lambda}(F+G)(x) \leq N_{\lambda / 2}(F)(x)+N_{\lambda / 2}(G)(x) .
$$

The advantage of $\mathcal{N}_{\lambda}$ is a pointwise comparison with the $q$-variation, namely $\mathcal{N}_{\lambda}(F)$ $\leq \lambda^{-q} V_{q}(F)^{q}$, and from (2) we see that also

$$
\lambda\left[N_{\lambda}(F)(x)\right]^{1 / q} \leq C_{q} V_{q}(F)(x)
$$

holds for any $q$, uniformly in $\lambda$, with $C_{q}=2^{1+1 / q}$. An argument of Bourgain allows us to often reverse this inequality in $L^{p}$ (see Lemma 2.1 below). For example, for a family of operators $\mathcal{A}=\left\{\mathcal{A}_{t}\right\}_{t} \in \mathfrak{I}$, uniform a priori bounds

$$
\left\|\lambda\left[N_{\lambda}(\mathcal{A} f)\right]^{1 / 2}\right\|_{L^{p}} \leq C_{p}\|f\|_{L^{p}}
$$

in an open range $p_{0}<p<p_{1}$ containing $p=2$, imply strong $q$-variational bounds for $q>2$ in the same range,

$$
\left\|V_{q}(\mathcal{A} f)\right\|_{L^{p}} \leq C_{p, q}\|f\|_{L^{p}}, \quad p_{0}<p<p_{1} .
$$

Our attention therefore will be concentrated on establishing uniform $L^{p}$ bounds for $\lambda\left[N_{\lambda}(\mathcal{A} f)\right]^{1 / 2}$ in some open range $p_{0}<p<p_{1}$. Such estimates give us quantitative information on the rate of convergence of $A_{t} f(x)$ as $t$ tends to zero.

Lépingle's inequality and applications. The main tool in proving variational inequalities is Lépingle's result 32 for a general bounded martingale sequence $\left\{f_{N}\right\}_{N=1}^{\infty}$; he proved $L^{p}, 1<p<\infty$ and weak type $(1,1)$ bounds for $V_{q}\left(f_{*}\right)$ whenever $q>2$. These estimates can fail for $q \leq 2$; see (37, [29]).

Simple proofs of Lépingle's inequality have been given by Pisier and Xu [36] and by Bourgain [5], by reducing matters to jump inequalities for the family $\left\{f_{N}\right\}$ (see \$2). As observed in [36] and [5], the jump inequalities for martingales follow from the pointwise estimate

$$
\lambda\left[\mathcal{N}_{\lambda}\left(f_{*}\right)(x)\right]^{1 / r} \leq\left(\sum_{k=1}^{\infty}\left|\mathfrak{F}_{k+1}(x)-\mathfrak{F}_{k}(x)\right|^{r}\right)^{1 / r}
$$

here $\mathfrak{F}_{k}(x)=f_{\tau_{k}(x)}(x)$ defines the stopped martingale constructed from the stopping times $\left\{\tau_{k}\right\}$ where $\tau_{k}(x)$ is the $k^{\text {th }}$ time the sequence $\left\{f_{k}(x)\right\}$ has jumped by at least $\lambda$. Then one can apply Burkholder's result (see [6]) for square functions of difference martingale sequences to the martingale $\left\{\mathfrak{F}_{k}\right\}$ in order to obtain the $L^{p}$ bounds, $1<p<\infty$,

$$
\left\|\lambda\left[\mathcal{N}_{\lambda}\left(f_{*}\right)\right]^{1 / r}\right\|_{p} \leq C_{p}\|f\|_{p}, \quad r \geq 2
$$

as well as weak type $(1,1)$ bounds

$$
\left|\left\{x: \lambda\left[\mathcal{N}_{\lambda}\left(f_{*}\right)\right]^{1 / r}>\alpha\right\}\right| \leq C \alpha^{-1}\|f\|_{1}, \quad r \geq 2 .
$$

By (2), we also have the $L^{p}$ and weak type $(1,1)$ bounds for $\lambda\left[N_{\lambda}\left(f_{*}\right)(x)\right]^{1 / r}, r \geq 2$, uniformly in $\lambda$.

In [5] Bourgain used Lépingle's result for the classical martingale generated by dyadic intervals to obtain similar variational estimates on $L^{2}(\mathbb{Z})$ for the averages 
$N^{-1} \sum_{n=1}^{N} f(m+n)$. These $L^{2}$ estimates have been extended to $L^{p}$ bounds for $1<$ $p<\infty$, and weak type $(1,1)$ bounds (see [26]). One can then transfer these estimates via Calderón's transference principle [7] to the general setting of a dynamical system $(X, \mu, T)$ and obtain $L^{p}$ (and weak type $\left.(1,1)\right)$ bounds for strong $q$-variations of the corresponding operators

$$
A_{N} f(x)=\frac{1}{N} \sum_{n=1}^{N} f\left(T^{n} x\right)
$$

where one is averaging over the orbit generated by the measure preserving automorphism $T$. This gives an alternative proof of Birkhoff's pointwise ergodic theorem since for any $x \in X$, if $V_{q}(\mathcal{A} f)(x)$ is finite for some finite $q$, then the limit of $A_{N} f(x)$ exists as $N$ tends to infinity. Traditionally one first controls a smaller object, the corresponding maximal function $M f(x)=\sup _{N>0}\left|A_{N} f(x)\right|$ in some $L^{p}$, reducing the pointwise result to proving convergence a.e. for some dense subclass of $L^{p}$ functions. Note the comparison $V_{\infty}(\mathcal{A} f)(x)=2 M f(x)$, yet $M f(x) \leq V_{\infty}(\mathcal{A} f)(x)+A_{t_{0}} f(x)$, for any $t_{0} \in \mathfrak{I}$. Establishing pointwise convergence a.e. for a dense class may not be straightforward in certain cases, and a variational $V_{q}(q<\infty)$ estimate shows almost everywhere convergence without this step, and, furthermore, gives some information about the rate of convergence.

Bourgain's work has inspired a number of authors to investigate variational bounds for other families of averaging operators, and also for families formed by truncating classical singular integral operators (see, e.g., [1, 9, , 10], 26], 27] and [28]). For example in [27, $L^{p}\left(\mathbb{R}^{d}\right), 1<p<\infty$, and weak type $(1,1)$ bounds were obtained for strong $q$-variations for the family of higher dimensional averages over Euclidean balls

$$
A_{t} f(x)=\frac{1}{\left|B_{t}(x)\right|} \int_{B_{t}(x)} f(y) d y
$$

thereby improving on the well-known estimates for the Hardy-Littlewood maximal function.

To establish strong $q$-variational estimates for a family of linear operators as in (6), the idea is to divide the analysis into short and long variations. Effectively, the short variations are defined in the same way except that the $\left\{t_{\ell}\right\}$ lie in some dyadic interval $\left[2^{j}, 2^{j+1}\right]$ whereas the long variations are computed over the sequence of dyadic numbers $\left\{2^{j}\right\}$. Known techniques from harmonic analysis are used to estimate the short variations whereas one compares the long variations with the variation along an appropriate martingale sequence. For example with respect to the $\left\{A_{t}\right\}$ in (6), the martingale sequence $\left\{f_{N}\right\}$ one takes is given by $f_{-N}=\mathbb{E}_{N} f=$ $\mathbb{E}\left(f \mid \mathcal{F}_{N}\right)$ where the $\sigma$-algebras $\mathcal{F}_{N}$ are generated from the usual ('half-open') dyadic cubes in $\mathbb{R}^{d}$; specifically,

$$
\mathbb{E}_{N} f(x)=\frac{1}{|Q|} \int_{Q} f
$$

where $Q$ is the unique dyadic interval of sidelength $2^{N}$ containing $x$. Then one can apply Lépingle's inequality to obtain strong $q$-variational estimates for $q>2$. The arguments in [37, 29] show that strong $q$-variational estimates with respect to the martingale sequence in (77) fail whenever $q \leq 2$ and hence there are no $L^{p}$ bounds for $V_{q}(\mathcal{A f}), \mathcal{A}=\left\{A_{t}\right\}$ with $A_{t}$ in (6), whenever $q \leq 2$. Thus, from now on we will restrict ourselves to the range $q>2$. 
In this paper we develop a general method which will allow us to obtain strong variational inequalities for a large class of averaging and truncated singular integral operators arising in harmonic analysis. For example, besides the family of linear operators in (6), we will consider more singular variants such as spherical means (see (17)) or averages along curves $\left(t, t^{a}\right)$ in the plane (here $a>1$ ). The latter operators exhibit some homogeneity with respect to nonisotropic dilations and our definition of the appropriate martingale has to reflect this dilation structure. Thus if $a$ is an integer we may work with the martingale sequence generated by rectangles (or generalized dyadic cubes) of the form $\left[j_{1} 2^{k},\left(j_{1}+1\right) 2^{k}\right) \times\left[j_{2} 2^{a k},\left(j_{2}+1\right) 2^{a k}\right)$ in the plane. There is no obvious definition of 'dyadic cubes' compatible with the dilation structure when $a \in \mathbb{R}^{+} \backslash \mathbb{Z}$. However there is a construction due to Christ [16] which shows that there is a family of suitable 'dyadic cubes' with all the necessary properties in the general setting of a space of homogeneous type. This allows one to define martingales which are appropriate for a general dilation structure

$$
t^{P}=\exp (P \log t)
$$

here $P$ is a real $d \times d$ matrix whose eigenvalues have positive real parts. Any regular quasi-norm $\rho$ which is homogeneous with respect to the dilations (i.e., $\left.\rho\left(t^{P} x\right)=t \rho(x), t>0\right)$ makes $\mathbb{R}^{d}$, together with Lebesgue measure and the quasimetric induced by $\rho$, into a space of homogeneous type (see $[50$ for the existence of a $\rho \in C^{\infty}\left(\mathbb{R}^{d} \backslash\{0\}\right)$ and other properties of general dilations $\left.t^{P}\right)$.

Jump inequalities - dyadic dilations. We formulate two main results for convolution operators whose formulation is motivated by the results on maximal operators of Duoandikoetxea and Rubio de Francia [21]. Below we shall discuss specific examples.

Let $\sigma$ be a compactly supported finite Borel measure on $\mathbb{R}^{d}$. We consider dilates $\sigma_{t}$ of $\sigma$ defined with respect to a group of dilations $\left\{t^{P}\right\}_{t>0}$ as in (8) with the dilate $\sigma_{t}$ being defined by

$$
\left\langle\sigma_{t}, f\right\rangle=\int f\left(t^{P} x\right) d \sigma
$$

We assume a weak regularity condition on $\sigma$ in terms of the Fourier transform:

$$
|\widehat{\sigma}(\xi)| \leq C|\xi|^{-b}, \quad \text { for some } b>0 .
$$

Under this assumption, bounds in $L^{p}\left(\mathbb{R}^{d}\right), 1<p \leq \infty$, were derived for the maximal operator defined by $M f(x)=\sup _{k \in \mathbb{Z}}\left|f * \sigma_{2^{k}}(x)\right|$. Moreover it is well known that these $L^{p}$ estimates can be extended to weak type $(1,1)$ bounds under the stronger hypothesis that $\sigma$ is absolutely continuous, with $L^{1}$ density $\Phi$ say, and

$$
\int_{\mathbb{R}^{d}}|\Phi(x+y)-\Phi(x)| d x \leq C|y|^{b}
$$

for some $b>0$ (see, e.g., [49, p. 72). Clearly (11) implies (10).

In this setting we extend these estimates as follows.

Theorem 1.1. Let $\mathfrak{A}=\left\{\mathfrak{A}_{k}\right\}$ where $\mathfrak{A}_{k} f(x)=f * \sigma_{2^{k}}(x)$ and $\sigma_{2^{k}}$ are as in (91).

(i) If $\sigma$ satisfies (10), then

$$
\left\|\lambda \sqrt{N_{\lambda}(\mathfrak{A} f)}\right\|_{L^{p}\left(\mathbb{R}^{d}\right)} \leq C_{p}\|f\|_{L^{p}\left(\mathbb{R}^{d}\right)}, \quad 1<p<\infty,
$$

uniformly in $\lambda>0$. Moreover $V_{q} \circ \mathfrak{A}$ is bounded on $L^{p}\left(\mathbb{R}^{d}\right)$ for $1<p<\infty, q>2$. 
(ii) If $\sigma$ satisfies (11), then in addition we have

$$
\left|\left\{x: \lambda \sqrt{N_{\lambda}(\mathfrak{A} f)}>\alpha\right\}\right| \leq \frac{C}{\alpha}\|f\|_{L^{1}\left(\mathbb{R}^{d}\right)},
$$

uniformly in $\lambda>0$. Moreover $V_{q} \circ \mathfrak{A}$ is of weak type $(1,1)$, for $q>2$.

We now state a theorem on truncated singular integral operators in the setting of convolution operators on $\mathbb{R}^{d}$ which are homogeneous with respect to a general group of dilations $\left\{t^{P}\right\}$.

We start with a compactly supported finite Borel measure $\nu$ on $\mathbb{R}^{d}$ with mean zero,

$$
\int d \nu=\widehat{\nu}(0)=0
$$

and consider the singular integral operator defined by $\operatorname{Tf}(x)=\sum_{k \in \mathbb{Z}} f * \nu_{2^{k}}(x)$, and its family of truncations $\mathcal{T}=\left\{T_{n}\right\}_{n \in \mathbb{Z}}$, where

$$
T_{n} f(x)=\sum_{n \leq k} f * \nu_{2^{k}}(x) .
$$

In 21], $L^{p}\left(\mathbb{R}^{d}\right)$ bounds $(1<p<\infty)$ were obtained for $T$ as well as for the associated maximal truncated operator, $T_{*} f(x)=\sup _{n}\left|\sum_{n \leq k} f * \nu_{2^{k}}(x)\right|$ if $\nu$ satisfies the weak regularity condition (10), and the same is true for the square function $g(f)(x)=\left(\sum_{k}\left|f * \nu_{2^{k}}(x)\right|^{2}\right)^{1 / 2}$. In both cases, these estimates can be extended to weak-type $(1,1)$ bounds if $\nu$ has an $L^{1}$ density satisfying (11); see, e.g., [22. In the following theorem we give bounds for the $\lambda$-jump function $N_{\lambda}(\mathcal{T} f)(x)$ of the discretely indexed family $\mathcal{T}$.

Theorem 1.2. Let $\mathcal{T}=\left\{T_{n}\right\}$ be as defined in (13), with $\nu$ satisfying (12).

(i) If $\nu$ satisfies (10), then

$$
\left\|\lambda \sqrt{N_{\lambda}(\mathcal{T} f)}\right\|_{L^{p}\left(\mathbb{R}^{d}\right)} \leq C_{p}\|f\|_{L^{p}\left(\mathbb{R}^{d}\right)}, \quad 1<p<\infty,
$$

uniformly in $\lambda>0$. Moreover $V_{q} \circ \mathcal{T}$ is bounded on $L^{p}\left(\mathbb{R}^{d}\right)$, for $1<p<\infty, q>2$.

(ii) If $\nu$ satisfies (11), then in addition we have

$$
\left|\left\{x: \lambda \sqrt{N_{\lambda}(\mathcal{T} f)(x)}>\alpha\right\}\right| \leq \frac{C}{\alpha}\|f\|_{L^{1}\left(\mathbb{R}^{d}\right)},
$$

uniformly in $\lambda>0$. Moreover $V_{q} \circ \mathcal{T}$ is of weak type $(1,1)$ if $q>2$.

Dyadic jump functions and short variation operators. In order to apply Theorems 1.1 and 1.2 we have to combine these results with certain square-function estimates. We now work with a family $\mathcal{A}=\left\{\mathcal{A}_{t}\right\}_{t \in \mathbb{R}^{+}}$and split the analysis of $N_{\lambda}(\mathcal{A} f)$ into short and long $\lambda$-jumps. Set

and let

$$
V_{\rho, j}(\mathcal{A} f)(x)=\left(\sup _{\substack{t_{1}<\ldots<t_{N} \\\left[t_{\ell}, t_{\ell+1}\right] \subset\left[2^{j}, 2^{j+1}\right]}} \sum_{\ell=1}^{N-1}\left|A_{t_{\ell+1}} f(x)-A_{t_{\ell}} f(x)\right|^{\rho}\right)^{1 / \rho}
$$

$$
S_{\rho}(\mathcal{A} f)(x)=\left(\sum_{j \in \mathbb{Z}}\left[V_{\rho, j}(\mathcal{A} f)(x)\right]^{\rho}\right)^{1 / \rho}
$$

be the short $\rho$-variation operator (we shall mostly use $\rho=2$ ). 
Define $N_{\lambda}^{\text {dyad }}(\mathcal{A} f)(x)$ to be the largest integer $N$ such that there is an increasing sequence of integers $j_{1}<k_{1} \leq j_{2}<k_{2} \leq \cdots \leq j_{N}<k_{N}$ over which

$$
\left|A_{2^{k_{\ell}}} f(x)-A_{2^{j \ell}} f(x)\right|>\lambda
$$

for each $\ell \in\{1, \ldots, N\}$. Thus $N_{\lambda}^{\text {dyad }}(\mathcal{A} f)$ is precisely the jump function for the dyadic families $\left\{A_{2^{n}}\right\}_{n \in \mathbb{Z}}$ treated in Theorems 1.1 and 1.2 .

One observes

\section{Lemma 1.3.}

$$
\lambda\left[N_{\lambda}(\mathcal{A} f)(x)\right]^{1 / \rho} \leq C\left(S_{\rho}(\mathcal{A} f)+\lambda\left[N_{\lambda / 3}^{\mathrm{dyad}}(\mathcal{A} f)(x)\right]^{1 / \rho}\right) .
$$

Proof. To prove this lemma we begin by fixing a sequence $s_{1}<t_{1} \leq \cdots \leq s_{N}<t_{N}$ such that $\left|A_{t_{\ell}} f(x)-A_{s_{\ell}} f(x)\right|>\lambda$ for each $\ell=1, \ldots, N$ and then separate the $\ell$ into two groups:

$$
J_{S}=\left\{\ell:\left[s_{\ell}, t_{\ell}\right] \subset\left[2^{j}, 2^{j+1}\right) \text { for some } j \in \mathbb{Z}\right\}
$$

and

First of all,

$$
J_{L}=\left\{\ell: s_{\ell}<2^{j} \leq t_{\ell} \text { for some } j \in \mathbb{Z}\right\}
$$

$$
\# J_{S} \leq \sum_{j \in \mathbb{Z}} N_{\lambda, j}(\mathcal{A} f)(x)
$$

where $N_{\lambda, j}$ is defined in exactly the same way as $N_{\lambda}$ except the sequence $s_{1}<t_{1} \leq$ $\cdots \leq s_{N}<t_{N}$ is required to lie entirely in the dyadic interval $\left[2^{j}, 2^{j+1}\right]$. For $\ell \in J_{L}$, choose $j_{\ell} \leq k_{\ell}$ so that $2^{j_{\ell}-1} \leq s_{\ell}<2^{j_{\ell}}$ and $2^{k_{\ell}} \leq t_{\ell}<2^{k_{\ell}+1}$. Then

$$
\begin{aligned}
\lambda & <\left|A_{t_{\ell}} f(x)-A_{s_{\ell}} f(x)\right| \\
& \leq\left|A_{t_{\ell}} f(x)-A_{2^{k_{\ell}}} f(x)\right|+\left|A_{2^{k_{\ell}}} f(x)-A_{2^{j_{\ell}}} f(x)\right|+\left|A_{2^{j_{\ell}}} f(x)-A_{s_{\ell}} f(x)\right|
\end{aligned}
$$

implies

$$
\# J_{L} \leq 2 \sum_{j \in \mathbb{Z}} N_{\lambda / 3, j}(\mathcal{A} f)(x)+N_{\lambda / 3}^{\mathrm{dyad}}(\mathcal{A} f)(x)
$$

and therefore

$$
N=\# J_{S}+\# J_{L} \leq 3 \sum_{j \in \mathbb{Z}} N_{\lambda / 3, j}(\mathcal{A} f)(x)+N_{\lambda / 3}^{\mathrm{dyad}}(\mathcal{A} f)(x) .
$$

Consequently we have

$$
\begin{aligned}
& \lambda {\left[N_{\lambda}(\mathcal{A} f)(x)\right]^{1 / \rho} \leq 3\left(\sum_{j \in \mathbb{Z}} \lambda^{\rho} N_{\lambda / 3, j}(\mathcal{A} f)(x)\right)^{1 / \rho}+\lambda\left[N_{\lambda / 3}^{\text {dyad }}(\mathcal{A} f)(x)\right]^{1 / \rho} } \\
& \leq C S_{\rho}(\mathcal{A} f)(x)+2 \lambda\left[N_{\lambda / 3}^{\text {dyad }}(\mathcal{A} f)(x)\right]^{1 / \rho}
\end{aligned}
$$

establishing (16).

Specific families of operators. We shall formulate some results for singular families of averages and singular integrals which follow from Theorems 1.1 and 1.2 and additional results for the short-variation operators.

We first consider the family of spherical means, defined by

$$
A_{t} f(x)=\int_{S^{d-1}} f(x-t y) d \sigma(y)
$$

here $d \sigma$ is the rotationally invariant measure on the unit sphere $S^{d-1}$, normalized to have mass 1 . The corresponding maximal operator is bounded on $L^{p}\left(\mathbb{R}^{d}\right)$ if 
and only if $p>d /(d-1)$. This was shown by Stein [48] when $d \geq 3$; the $d=2$ case was established later by Bourgain [4]. In dimensions $d \geq 3$, Bourgain [3] also established a restricted weak type inequality for the endpoint $p=d /(d-1)$; i.e., the maximal operator maps the Lorentz space $L^{d /(d-1), 1}$ to $L^{d /(d-1), \infty}$. The latter result fails in two dimensions (see [43]). Here we prove the following variational and jump estimates for the family of spherical means.

Theorem 1.4. Suppose $d \geq 2$ and $\mathcal{A}=\left\{A_{t}\right\}$ are the spherical means defined in (17).

(i) The a priori estimates

$$
\left\|V_{q}(\mathcal{A} f)\right\|_{L^{p}\left(\mathbb{R}^{d}\right)} \leq C_{p, q}\|f\|_{L^{p}\left(\mathbb{R}^{d}\right)}
$$

hold whenever $d /(d-1)<p \leq 2 d$ and $q>2$. Furthermore for the range $p>2 d$, if $q>p / d$, then (18) holds and conversely, if (18) holds, then we necessarily have $q \geq p / d$.

(ii) Suppose $d \geq 3$. Then for $d /(d-1)<p<2 d$,

$$
\left\|\lambda \sqrt{N_{\lambda}(\mathcal{A} f)}\right\|_{p} \leq C_{p}\|f\|_{p} .
$$

(iii) Suppose $d \geq 3$. Then there is the restricted weak-type inequality

$$
\left\|\lambda \sqrt{N_{\lambda}(\mathcal{A} f)}\right\|_{L^{\frac{d}{d-1}, \infty}} \leq C\|f\|_{L^{\frac{d}{d-1}, 1}} .
$$

Moreover, if $q>2$, then $V_{q} \circ \mathcal{A}$ maps $L^{\frac{d}{d-1}, 1}$ to $L^{\frac{d}{d-1}, \infty}$.

Remark. The maximal operator bounds of Stein and Bourgain were transferred to the setting of a dynamical system in 25] and [31] where pointwise convergence results were established. These results follow at once from the strong $q$-variational estimates in Theorem 1.4

Another family of singular averaging operators is formed by taking averages over curves in $\mathbb{R}^{2}$,

$$
A_{t} f\left(x_{1}, x_{2}\right)=\frac{1}{t} \int_{0}^{t} f\left(x_{1}-s, x_{2}-s^{a}\right) d s .
$$

This and related families have been extensively studied (see, e.g., [50]). For instance, it is known that the associated maximal operator $M f(x)=\sup _{t>0}\left|A_{t} f(x)\right|$ is bounded on all $L^{p}\left(\mathbb{R}^{2}\right), 1<p \leq \infty$, 35]; however it remains an open question whether weak type $(1,1)$ bounds hold (see [17, [4] for results near $L^{1}$ ). We extend these estimates in the following way.

Theorem 1.5. Let $\mathcal{A}=\left\{A_{t}\right\}$ where $A_{t}$ is defined in (21). Then for $1<p<\infty$,

$$
\left\|\lambda \sqrt{N_{\lambda}(\mathcal{A f})}\right\|_{p} \leq C_{p}\|f\|_{L^{p}\left(\mathbb{R}^{2}\right)}
$$

moreover $V_{q} \circ \mathcal{A}$ is bounded on $L^{p}$ for $q>2$.

Remark. This result can be generalized to averaging operators along any $k$-dimensional surface in $\mathbb{R}^{d}$ which possesses a certain amount of curvature; namely, every hyperplane has a finite order of contact with the surface. Specifically one can replace the curve $\left\{\left(s, s^{a}\right)\right\}$ in the plane with a $k$-surface in $\mathbb{R}^{d}$ parameterized by $\Phi(s)=$ $\left(s^{\alpha_{1}}, \ldots, s^{\alpha_{d}}\right)$, where $s \in \mathbb{R}^{k}$ and the monomials defining the components of $\Phi$ are distinct. One considers the family of averages $A_{t} f(x)=\frac{1}{\varpi_{k} t^{k}} \int_{|s| \leq t} f(x-\Phi(s)) d s$; here $\varpi_{k}$ denotes the volume of the unit ball in $\mathbb{R}^{k}$ and proves the above jump 
inequalities for this family. See [49] for the related arguments for the maximal operator.

We now turn to families of operators defined by truncations of classical singular operators. Let $\Omega \in L^{1}\left(S^{d-1}\right)$ so that the cancellation condition

$$
\int_{S^{d-1}} \Omega(\theta) d \sigma(\theta)=0
$$

holds, and define the family $\mathcal{T}=\left\{T_{t}\right\}$ by

$$
T_{t} f(x)=\text { p.v. } \int_{|y|>t} \Omega(y /|y|)|y|^{-d} f(x-y) d y .
$$

It is known that under the condition $\Omega \in L \log L\left(S^{d-1}\right)$ the operators $T_{t}$ are uniformly bounded (see 8$]$ ); moreover, variational $L^{p}\left(V_{q}\right)$ bounds and consequently bounds for $\lambda\left(N_{\lambda}(\mathcal{T} f)\right)^{1 / q}, q>2$, have been obtained in [9, 10. Here we settle the jump inequalities for the endpoint case $q=2$ which had remained conjectural in these papers.

Theorem 1.6. Let $\Omega, \mathcal{T}$ be as in (22), (23). Suppose that $\Omega \in L^{r}\left(S^{d-1}\right)$ for some $r>1$. Then for $1<p<\infty$,

$$
\left\|\lambda \sqrt{N_{\lambda}(\mathcal{T} f)}\right\|_{p} \leq C_{p}\|f\|_{p}
$$

Remark. By extending arguments in [10] one can prove for the short variation $S_{2}(\mathcal{T} f)$ a weak type $(1,1)$ inequality provided that if $d \geq 2$, then $\Omega$ belongs to the Sobolev class $L_{\alpha}^{1}\left(S^{d-1}\right)$ for some $\alpha>0$. Then by the weak-type $(1,1)$ version of Theorem 1.2 one also gets a weak type $(1,1)$ endpoint inequality for $\lambda \sqrt{N_{\lambda}(\mathcal{T} f)}$. In one dimension we get a weak type $(1,1)$ bound for the truncation of the Hilbert transforms $T_{t} f(x)=\int_{|y|>t} f(x-y) y^{-1} d y$. This weak type inequality implies

$$
\left|\left\{x: N_{\lambda}(\mathcal{T} f)>n\right\}\right| \leq C n^{-1 / 2} \lambda^{-1}\|f\|_{1}
$$

for $\lambda>0, n=1,2, \ldots$. We note that [9, 10] contain similar weak-type bounds for regular singular integrals but with $n^{-1 / 2}$ replaced by $n^{\varepsilon-1 / 2}, \varepsilon>0$.

One can ask whether these jump inequalities hold without the additional regularity assumption on $\Omega$. It is known [42] (see also [18, 24], [52]) that the operators $\mathcal{T}$ satisfy uniform weak-type $(1,1)$ estimates if $\Omega \in L \log L$. However weak type $(1,1)$ inequalities for the jumps and $V_{q}$ variations and even for the maximal function $\sup _{t>0}\left|T_{t} f\right|$ remain open for rough $\Omega$.

We conclude by formulating a theorem for Hilbert transforms along the plane curves $\left\{\left(s, s^{a}\right)\right\}$. Define $\mathcal{H}=\left\{H_{t}\right\}$ by

$$
H_{t} f\left(x_{1}, x_{2}\right)=\int_{|y|>t} f\left(x_{1}-s, x_{2}-s^{a}\right) \frac{d s}{s} .
$$

It is well known that the maximal function $\sup _{t>0}\left|H_{t} f(x)\right|$ defines a bounded operator on $L^{p}\left(\mathbb{R}^{d}\right)$; see [50, 21].

Theorem 1.7. For $1<p<\infty$ we have

$$
\left\|\lambda \sqrt{N_{\lambda}(\mathcal{H} f)}\right\|_{p} \leq C_{p}\|f\|_{L^{p}\left(\mathbb{R}^{2}\right)} ;
$$

moreover $V_{q} \circ \mathcal{H}$ is bounded on $L^{p}, 1<p<\infty$. 
In the next section we discuss an argument by Bourgain which shows how to obtain strong $q$-variational bounds from estimates on the corresponding $\lambda$-jump function. In $\$ 3$ we discuss Christ's construction of dyadic cubes in the setting of a space of homogeneous type and use this in the proofs of Theorems 1.1 and 1.2, given in 9 and $\$ 5$ respectively. In $\$ 6$ we discuss how harmonic analysis techniques can be used in the study of the short 2-variation operator. Then in $\$ 7$ we give the proofs of the results for averages and singular integrals associated to a parabola and in 88 we discuss spherical means.

\section{From $\lambda$-Jumps to $q$-VARiations}

We present here a slight generalization of Bourgain's argument in [5] reducing variational inequalities to jump inequalities. Let $(X, d \mu)$ be a measure space and consider a family of linear operators $\mathcal{T}=\left\{T_{t}\right\}$, where $t$ is taken from a finite collection $\mathcal{F}$ of positive numbers and $T_{t}$ maps $L^{p}(X)$ functions to measurable functions.

Recall the definition of $\lambda$-jumps $N_{\lambda}(\mathcal{T} f)(x)$ and $q$-variation $V_{q}(\mathcal{T} f)(x)$ and define for an integer $m$,

$$
V_{q}^{m}(\mathcal{T} f)(x)=\sup _{2^{m} \leq\left|T_{t_{i}} f(x)-T_{t_{i+1}} f(x)\right| \leq 2^{m+1}}\left(\sum_{i}\left|T_{t_{i}} f(x)-T_{t_{i+1}} f(x)\right|^{q}\right)^{1 / q}
$$

so that now the supremum in the definition of $V_{q}(\mathcal{T} f)(x)$ is restricted to those $t_{i}$ where $2^{m} \leq\left|T_{t_{i}} f(x)-T_{t_{i+1}} f(x)\right| \leq 2^{m+1}$ (we define $V_{q}^{m}(\mathcal{T} f)(x)=0$ if $\mid T_{t} f(x)-$ $T_{t^{\prime}} f(x) \mid \notin\left[2^{m}, 2^{m+1}\right]$ for any choice of $t$ and $\left.t^{\prime}\right)$.

In what follows we shall use the obvious inequalities

$$
\left|V_{s}^{m}(\mathcal{T} f)(x)\right|^{s} \leq 2^{m s} N_{2^{m}}(\mathcal{T} f)(x)
$$

for $s>0$ and

$$
V_{s}(\mathcal{T} f)(x) \leq\left(\sum_{m \in \mathbb{Z}}\left|V_{s}^{m}(\mathcal{T} f)(x)\right|^{s}\right)^{1 / s}
$$

moreover $V_{s}(\mathcal{T} f)(x)$ increases when $s$ decreases.

Lemma 2.1. Suppose that $p_{0}<\rho<p_{1}$ and that for $p_{0}<p<p_{1}$ the inequality

$$
\sup _{\lambda>0}\left\|\lambda\left[N_{\lambda}(\mathcal{T} f)\right]^{1 / \rho}\right\|_{p} \leq C\|f\|_{p}
$$

holds for all $f$ in (a dense subset of) $L^{p}$. Then we have for $\rho<q$,

$$
\left\|V_{q}(\mathcal{T} f)\right\|_{p} \leq C(p, q)\|f\|_{p}
$$

for $f \in L^{p}, p_{0}<p<p_{1}$. In particular, if $p$ is restricted to a compact subinterval $J=\left[p_{L}, p_{R}\right]$ of $\left(p_{0}, p_{1}\right)$ we have the estimate $C(p, q) \leq C_{J}(q-\rho)^{-1}$ for $p \in J$, $q>\rho$.

Proof. It suffices to prove a restricted strong type estimate, i.e.,

$$
\left\|V_{q}\left(\mathcal{T} \chi_{A}\right)\right\|_{p} \leq C(p, q)|A|^{1 / p}
$$

for characteristic functions of measurable sets $A$, with finite measure (see [51], ch. V.3).

We estimate

$$
\begin{aligned}
\left\|V_{q}(\mathcal{T} f)\right\|_{p} & \leq\left\|\left(\sum_{m \leq 0}\left|V_{q}^{m}(\mathcal{T} f)\right|^{q}\right)^{1 / q}\right\|_{p}+\left\|\left(\sum_{m \geq 0}\left|V_{q}^{m}(\mathcal{T} f)\right|^{q}\right)^{1 / q}\right\|_{p} \\
& :=I+I I .
\end{aligned}
$$


Note that it suffices to obtain the bound for $q>\rho$ and $q$ close to $\rho$ since the expressions in $q$ increase if $q$ decreases.

In what follows we abbreviate $N_{2^{m}}\left(\mathcal{T} \chi_{A}\right)(x)=N_{m}(x)$.

Estimation of $I$. Let $r=p \rho / q$. We may assume that $q>\rho$ is so small that for $p \in J$ we have $r \geq p_{0}+\left(p_{L}-p_{0}\right) / 2>p_{0}$. Now by (26) we have

$$
I \leq\left(\int\left(\sum_{m \leq 0} 2^{m q} N_{m}(x)\right)^{p / q} d x\right)^{1 / p} .
$$

We distinguish the cases $p \geq q$ and $p<q$.

If $p \geq q$ we use Minkowski's inequality (for the exponent $p / q=r / \rho$ ) to dominate $I$ by

$$
\begin{aligned}
I & \leq\left(\int\left(\sum_{m \leq 0} 2^{m(q-\rho)} N_{m}(x) 2^{m \rho}\right)^{p / q} d x\right)^{1 / p} \\
& \leq\left(\sum_{m \leq 0} 2^{m(q-\rho)}\left(\int N_{m}(x)^{p / q} 2^{m \rho p / q} d x\right)^{q / p}\right)^{1 / q} \\
& =\left(\sum_{m \leq 0} 2^{m(q-\rho)}\left\|N_{m}^{1 / \rho} 2^{m}\right\|_{r}^{\rho}\right)^{1 / q} \leq C|q-\rho|^{-1} \sup _{m}\left\{\left\|N_{m}^{1 / \rho} 2^{m}\right\|_{r}^{r / p}\right\} \\
& \leq C^{\prime}|q-\rho|^{-1}|A|^{1 / p} .
\end{aligned}
$$

Next we consider the case $p<q$ and apply the triangle inequality $(a+b)^{\gamma} \leq a^{\gamma}+b^{\gamma}$ for $\gamma=p / q<1$. Now $I$ is dominated by

$$
\left(\int \sum_{m \leq 0} 2^{m p} N_{m}(x)^{p / q} d x\right)^{1 / p}=\left(\sum_{m \leq 0} 2^{m p(1-\rho / q)}\left\|N_{m}^{1 / \rho} 2^{m}\right\|_{r}^{r}\right)^{1 / p}
$$

which is again estimated by $C|q-\rho|^{-1} \sup _{m}\left\{\left\|N_{m}^{1 / \rho} 2^{m}\right\|_{r}^{r / p}\right\}$ and thus by $C^{\prime}|q-\rho|^{-1}|A|^{1 / p}$.

Estimation of $I I$. We now assume that $p<r:=p_{1}-\left(p_{1}-p_{R}\right) / 2$ and set $s=p \rho / r$. In particular $s<\rho<q$ and $p_{0}<p<r<p_{1}$. Again we distinguish two cases, $p \geq s$ and $p<s$. If $p \geq s$, we use Minkowski's inequality and (26) to bound II by

$$
\begin{aligned}
I I & \leq\left\|\left(\sum_{m \geq 0}\left|V_{s}^{m}(\mathcal{T} f)(x)\right|^{s}\right)^{1 / s}\right\|_{p} \\
& \leq\left(\int\left(\sum_{m \geq 0} 2^{m s} N_{m}(x)\right)^{p / s} d x\right)^{1 / p} \\
& \leq\left(\sum_{m \geq 0} 2^{m p}\left(\int N_{m}(x)^{p / s}\right)^{s / p} d x\right)^{1 / s} \\
& =\left(\sum_{m \geq 0} 2^{m s(1-r / p)}\left(\int 2^{m r} N_{m}(x)^{r / \rho}\right)^{\rho / r} d x\right)^{1 / s} .
\end{aligned}
$$

Since $r>p$ we can dominate this by a constant times $|A|^{\rho /(r s)}=|A|^{1 / p}$. 
If $p<s$ we use the triangle inequality $(a+b)^{\gamma} \leq a^{\gamma}+b^{\gamma}$ for $\gamma=p / s<1$ and dominate $I I$ by

$$
\begin{aligned}
& \left(\int \sum_{m \geq 0} 2^{m p} N_{m}^{p / s} d x\right)^{1 / p}=\left(\sum_{m \geq 0} 2^{m(p-r)} \int 2^{m r} N_{m}^{r / \rho} d x\right)^{1 / p} \\
& \leq\left(\sum_{m \geq 0} 2^{m(p-r)}|A|\right)^{1 / p} \leq C_{J}|A|^{1 / p}
\end{aligned}
$$

\section{Dyadic Cubes in spaces of homogeneous type}

In the proofs of Theorems 1.1 and 1.2 we will compare the $\lambda$-jump functions to the $\lambda$-jump function associated to a martingale sequence generated by 'dyadic cubes' adapted to the dilation group $\left\{t^{P}\right\}$. More precisely for each integer $k \in \mathbb{Z}$, we shall partition $\mathbb{R}^{d}=\bigcup Q_{\alpha}^{k}$ such that for each $\alpha$, the diameter of $Q_{\alpha}^{k}$ (measured with respect to a homogeneous quasi-norm $\rho$ which we fix once and for all) is roughly $A^{k}$ for a fixed constant $A>1$ depending only on the dilation group $\left\{t^{P}\right\}$. Moreover, the family $\left\{Q_{\alpha}^{k}\right\}$ behaves like a collection of dyadic cubes in that if $\ell \leq k$, either $Q_{\beta}^{\ell} \subset Q_{\alpha}^{k}$ or $Q_{\beta}^{\ell} \cap Q_{\alpha}^{k}=\emptyset$. Also for each $Q_{\beta}^{\ell}$ and $\ell<k$, there is a unique $\alpha$ such that $Q_{\beta}^{\ell} \subset Q_{\alpha}^{k}$. The martingale sequence is of the form $\mathbb{E}_{k} f=\mathbb{E}\left(f \mid \mathcal{F}_{k}\right)$ where $\mathcal{F}_{k}$ is the $\sigma$-algebra generated by the 'cubes' $\left\{Q_{\alpha}^{k}\right\}$. That is,

$$
\mathbb{E}_{k} f(x)=\frac{1}{\left|Q_{\alpha}^{k}\right|} \int_{Q_{\alpha}^{k}} f,
$$

where $Q_{\alpha}^{k}$ is the set in the partition $\mathbb{R}^{d}=\bigcup Q_{\alpha}^{k}$ containing $x$.

The existence of the generalized dyadic cubes was shown by Christ [16] who constructed them in the general setting of a space of homogeneous type $X$. Here $X$ is a space with a quasi-metric $d$; i.e., $d$ satisfies all the properties of a metric except that the triangle inequality is relaxed to $d(x, y) \leq C_{0}[d(x, z)+d(z, y)]$ for some $C_{0} \geq 1$ but we still require the associated balls $B(x, r)$ defined by $d$ to be open sets. Furthermore $X$ is equipped with a positive finite Borel measure $\mu$ possessing the doubling condition

$$
\mu(B(x, 2 r)) \leq C_{1} \mu(B(x, r))
$$

for all $x \in X, r>0$. It is well known that a large part of the theory of singular integrals and maximal functions can be carried out in such a setting $(X, d, \mu)$; see, e.g., 49. In particular, the maximal operator

$$
\mathcal{M} f(x)=\sup _{r>0} \frac{1}{\mu(B(x, r))} \int_{B(x, r)}|f(y)| d \mu(y)
$$

is bounded on all $L^{p}(X, \mu), 1<p \leq \infty$ and satisfies weak type $(1,1)$ bounds. In this setting we quote the following result of Christ.

Lemma 3.1 ([16]). There exists a collection of open sets $\left\{Q_{\alpha}^{k}: k \in \mathbb{Z}, \alpha \in I_{k}\right\}$ and constants $A>1, a_{0}, \eta>0$ and $C_{2}, C_{3}<\infty$ such that

(i) $\mu\left(X \backslash \bigcup_{\alpha} Q_{\alpha}^{k}\right)=0$, for all $k \in \mathbb{Z}$;

(ii) if $\ell \leq k$, then either $Q_{\beta}^{\ell} \subset Q_{\alpha}^{k}$ or $Q_{\beta}^{\ell} \cap Q_{\alpha}^{k}=\emptyset$;

(iii) for each $(\ell, \beta)$ and $\ell \leq k$, there exists a unique $\alpha$ such that $Q_{\beta}^{\ell} \subset Q_{\alpha}^{k}$;

(iv) each $Q_{\alpha}^{k}$ contains some ball $B\left(z_{\alpha}^{k}, a_{0} A^{k}\right)$ and diameter $\left(Q_{\alpha}^{k}\right) \leq C_{2} A^{k}$;

(v) $\mu\left(\left\{x \in Q_{\alpha}^{k}: d\left(x, X \backslash Q_{\alpha}^{k}\right) \leq t A^{k}\right\}\right) \leq C_{3} t^{\eta} \mu\left(Q_{\alpha}^{k}\right), \forall k, \alpha, \forall t>0$. 
Remarks. (i) We will use Lemma 3.1 when $X=\mathbb{R}^{d}, \mu$ is Lebesgue measure and the quasi-metric is induced by a quasi-norm $\rho$ which is homogeneous with respect to the group of dilations $\left\{t^{P}\right\}, \rho\left(t^{P} x\right)=t \rho(x)$. In this case since each cube $Q_{\alpha}^{k}$ contains and is contained in a ball of radius about $A^{k}$, and since the quasi-metric is translation-invariant, we see that the measure of $Q_{\alpha}^{k}$ is roughly $A^{(\operatorname{tr} P) k}$ for each $\alpha$.

(ii) Property (v) in Lemma 3.1 says that the (characteristic functions of) dyadic cubes possess some smoothness, as not too much mass accumulates near the boundary. This will be essential for us in our analysis.

(iii) David ([20]) had previously constructed dyadic cubes with all the properties (i)-(v) in the more restrictive setting of an Ahlfors regular measure-metric space where one can take $A=2$ in Lemma 3.1. For our purposes we need the more general construction.

As an illustration of how we will use property (v) in Lemma 3.1 we prove a basic $L^{2}$ estimate with respect to the group of dilations $\left\{t^{P}\right\}$ in $\mathbb{R}^{d}$ (and associated homogeneous quasi-norm $\rho$ ). We emphasize that very similar arguments are in [26] and also in 23. Lemma 3.1 gives us a family $\left\{Q_{\alpha}^{k}\right\}$ of dyadic cubes which have diameter $\leq C_{2} A^{k}$. For each integer $k$, let $\ell=\ell(k)$ be the integer such that $2^{\ell} \leq A^{k}<2^{\ell+1}$. Let $\phi$ be a Schwartz function with $\int \phi=1$, and set $\phi_{\ell}(x)=$ $2^{-(\operatorname{tr} P) \ell} \phi\left(2^{-\ell P} x\right)$ so that $\left\|\phi_{\ell}\right\|_{1}=\|\phi\|_{1}$.

Lemma 3.2. Set $\mathbb{D}_{k} f=\mathbb{E}_{k} f-\mathbb{E}_{k-1} f$, where $\left\{\mathbb{E}_{k} f\right\}$ is the martingale sequence defined in (27). Then

$$
\left\|\phi_{\ell(k+m)} * \mathbb{D}_{m} f-\mathbb{E}_{k+m}\left(\mathbb{D}_{m} f\right)\right\|_{2} \leq C 2^{-\delta|k|}\left\|\mathbb{D}_{m} f\right\|_{2}
$$

for some $\delta>0$.

Proof. Uniform $L^{2}$ bounds without the extra decay in $k$ clearly hold, so we may assume $|k| \gg 1$. We consider two cases: $k$ positive and $k$ negative.

When $k \geq 0$ we have $\mathbb{E}_{k+m}\left(\mathbb{E}_{m} f\right)=\mathbb{E}_{k+m} f$ from properties (i)-(iii) in Lemma 3.1, and hence $\mathbb{E}_{k+m}\left(\mathbb{D}_{m} f\right)=0$. In particular for all $\alpha$ we have $\int_{Q_{\alpha}^{m}} \mathbb{D}_{m} f(y) d y=0$, and so

$$
\phi_{\ell(k+m)} * \mathbb{D}_{m} f(x)=\sum_{\alpha} \int_{Q_{\alpha}^{m}}\left[\phi_{\ell(k+m)}(x-y)-\phi_{\ell(k+m)}\left(x-z_{\alpha}^{m}\right)\right] \mathbb{D}_{m}(y) d y .
$$

By the mean value theorem we estimate

$$
\begin{gathered}
\left|\phi_{\ell(k+m)}(x-y)-\phi_{\ell(k+m)}\left(x-z_{\alpha}^{m}\right)\right| \\
\leq C_{N}\left[\rho\left(2^{-(\ell(k+m)) P}\left(y-z_{\alpha}^{m}\right)\right)\right]^{\delta} \frac{2^{-(\ell(k+m)) t r P}}{\left[1+2^{-(\ell(k+m))} \rho(x-y)\right]^{N}},
\end{gathered}
$$

for any $N$. Here we have used a basic relationship between the Euclidean norm and $\rho$

$$
\rho(x) \leq|x|^{\alpha}, \rho(x) \geq 1, \text { and } \rho(x) \leq|x|^{\delta}, \rho(x) \leq 1
$$

for some $\alpha, \delta>0$. Therefore, as in the Euclidean case,

$$
\begin{aligned}
\left|\phi_{\ell(k+m)} * \mathbb{D}_{m} f(x)\right| & \leq C_{N} A^{-\delta k} \int_{\mathbb{R}^{d}} \frac{2^{-(\ell(k+m)) \operatorname{tr} P}}{\left[1+2^{-(\ell(k+m))} \rho(x-y)\right]^{N}}\left|\mathbb{D}_{m} f(y)\right| d y \\
& \leq C 2^{-\delta^{\prime} k} \mathcal{M}\left(\mathbb{D}_{m} f\right)(x),
\end{aligned}
$$


and so

$$
\begin{gathered}
\left\|\phi_{\ell(k+m)} * \mathbb{D}_{m} f-\mathbb{E}_{k+m}\left(\mathbb{D}_{m} f\right)\right\|_{2}=\left\|\phi_{\ell(k+m)} * \mathbb{D}_{m} f\right\|_{2} \\
\leq C 2^{-\delta^{\prime} k}\left\|\mathcal{M}\left(\mathbb{D}_{m} f\right)\right\|_{2} \leq C 2^{-\delta^{\prime} k}\left\|\mathbb{D}_{m} f\right\|_{2}
\end{gathered}
$$

establishing (28) in this case.

When $k \leq 0, \mathbb{E}_{k+m}\left(\mathbb{E}_{m} f\right)=\mathbb{E}_{m} f$ and so $\mathbb{E}_{k+m}\left(\mathbb{D}_{m} f\right)=\mathbb{D}_{m} f$. Thus

$$
\begin{aligned}
& \phi_{\ell(k+m)} * \mathbb{D}_{m} f(x)-\mathbb{E}_{k+m}\left(\mathbb{D}_{m} f\right)(x)=\int_{\mathbb{R}^{d}}\left[\mathbb{D}_{m} f(x-y)-\mathbb{D}_{m} f(x)\right] \phi_{\ell(k+m)}(y) d y \\
& =\int_{\rho(y) \leq 2^{\ell(k+m)}}\left[\mathbb{D}_{m} f(x-y)-\mathbb{D}_{m} f(x)\right] \phi_{\ell(k+m)}(y) d y \\
& \quad+\sum_{n \geq 1} \int_{E_{k, m, n}}\left[\mathbb{D}_{m} f(x-y)-\mathbb{D}_{m} f(x)\right] \phi_{\ell(k+m)}(y) d y \\
& :=I_{0}(x)+\sum_{n \geq 1} I_{n}(x),
\end{aligned}
$$

where $E_{k, m, n}=\left\{y: 2^{\ell(k+m+n-1)} \leq \rho(y) \leq 2^{\ell(k+m+n)}\right\}$.

We first treat $I_{n}(x)$ for $n \geq \epsilon|k|$ where we use the bound

$$
\begin{aligned}
\left|\phi_{\ell(k+m)}(y)\right| & \leq C_{N} 2^{-(\ell(k+m)) \operatorname{tr} P}\left[\left(2^{-(\ell(k+m))} \rho(y)\right]^{-N}\right. \\
& \leq C_{N} A^{-N n} 2^{-(\ell(k+m)) \operatorname{tr} P}
\end{aligned}
$$

on $E_{k, m, n}$, for any $N>0$, and Minkowski's inequality to estimate

$$
\left\|I_{n}\right\|_{2} \leq C_{N} 2^{-N^{\prime} n}\left\|\mathbb{D}_{m} f\right\|_{2} \int_{E_{k, m, n}} 2^{-(\ell(k+m)) \operatorname{tr} P} d y \leq C_{N} 2^{-\left(N^{\prime}-\operatorname{tr} P\right) n}\left\|\mathbb{D}_{m} f\right\|_{2} .
$$

Taking $N^{\prime}>\operatorname{tr} P$ allows us to sum in $n$ so that

$$
\left\|\sum_{n \geq \epsilon|k|} I_{n}\right\|_{2} \leq C 2^{-\epsilon^{\prime}|k|}\left\|\mathbb{D}_{m} f\right\|_{2} .
$$

For each $0 \leq n \leq \epsilon|k|$ and $Q_{\alpha}^{m-1}$, we consider those $x \in Q_{\alpha}^{m-1}$ such that $\operatorname{dist}\left(x, \mathbb{R}^{d} \backslash Q_{\alpha}^{m-1}\right) \geq A^{m+n+k}$ and observe that for $\rho(y) \leq 2^{\ell(k+m+n)}$, both $x$ and $x-y$ remain in $Q_{\alpha}^{m-1}$ and so $\mathbb{D}_{m} f(x-y)$ and $\mathbb{D}_{m} f(x)$ agree. Hence

$$
\begin{aligned}
\left\|I_{n}\right\|_{2}^{2} & =\sum_{\alpha} \int_{\left\{x \in Q_{\alpha}^{m-1}: \operatorname{dist}\left(x, \mathbb{R}^{d} \backslash Q_{\alpha}^{m-1}\right) \leq A^{m+n+k}\right\}}\left|I_{n}(x)\right|^{2} d x \\
& \leq \sum_{\alpha} \sup _{x \in Q_{\alpha}^{m-1}}\left|I_{n}(x)\right|^{2}\left|\left\{x \in Q_{\alpha}^{m-1}: \operatorname{dist}\left(x, \mathbb{R}^{d} \backslash Q_{\alpha}^{m-1}\right) \leq A^{m+n+k}\right\}\right| \\
& \leq C 2^{-\eta|k|} \sum_{\alpha}\left|Q_{\alpha}^{m-1}\right| \sup _{x \in Q_{\alpha}^{m-1}}\left|I_{n}(x)\right|^{2}
\end{aligned}
$$

for some $\eta>0$ by property (v) in Lemma 3.1. However for $x \in Q_{\alpha}^{m-1}$,

$$
\left|I_{n}(x)\right| \leq C \sup _{x \in B\left(z_{\alpha}^{m-1}, C^{\prime} A^{m}\right)}\left|\mathbb{D}_{m} f(x)\right|
$$

for some $C^{\prime}$. Since the measure of any ball of radius $A^{m}$ is about $A^{(\operatorname{tr} P) m}$ we see that the cardinality of

$$
N_{m, \alpha}=\left\{\beta: Q_{\beta}^{m-1} \cap B\left(z_{\alpha}^{m-1}, C^{\prime} A^{m}\right) \neq \emptyset\right\}
$$


is uniformly bounded as well as the number, for each fixed $\beta$, of $\alpha$ such that $\beta \in$ $N_{m, \alpha}$. Hence

$$
\begin{aligned}
\left\|I_{n}\right\|_{2}^{2} & \leq C 2^{-\eta|k|} \sum_{\alpha} \sum_{\beta \in N_{m, \alpha}} \int_{Q_{\beta}^{m-1}}\left|\mathbb{D}_{m} f(x)\right|^{2} d x \\
& \leq C^{\prime} 2^{-\eta|k|} \sum_{\beta} \int_{Q_{\beta}^{m-1}}\left|\mathbb{D}_{m} f(x)\right|^{2} d x \\
& =C^{\prime} 2^{-\eta|k|}\left\|\mathbb{D}_{m} f\right\|_{2}^{2}
\end{aligned}
$$

which implies

$$
\left\|\sum_{0 \leq n \leq \epsilon|k|} I_{n}\right\|_{2} \leq C 2^{-\delta|k|}\left\|\mathbb{D}_{m} f\right\|_{2}
$$

for some $\delta>0$. Together with (31), this completes the proof of the lemma.

\section{Proof of Theorem 1.1}

We are trying to establish uniform $L^{p}\left(\mathbb{R}^{d}\right)$ bounds for the $\lambda$-jump operator $\lambda\left[N_{\lambda}(\mathfrak{A} f)\right]^{1 / 2}$ for the discretely indexed family $\mathfrak{A} f=\left\{f * \sigma_{2^{k}}\right\}_{k \in \mathbb{Z}}$ of convolution operators on $\mathbb{R}^{d}$; here $\sigma_{t}$ is the $t^{P}$-dilate of a compactly supported finite Borel measure $\sigma$ satisfying either regularity condition (10) or condition (11). We may assume $\int d \sigma \neq 0$ since $\lambda^{2} N_{\lambda}(\mathfrak{A} f)(x)$ is always pointwise dominated by the square function $\sum_{k}\left|f * \sigma_{2^{k}}(x)\right|^{2}$, and if $\widehat{\sigma}(0)=0$, known bounds from 21] apply. Therefore we may normalize $\sigma$ so that $\int d \sigma=1$. Let $\phi$ be a smooth function with compact support such that $\int \phi=1$ and decompose $\sigma=\phi * \sigma+\left(\delta_{0}-\phi\right) * \sigma$ where $\delta_{0}$ is the Dirac mass at 0 . This in turn decomposes $\mathfrak{A}$ into low and high frequency families $\mathcal{L}=\left\{\mathcal{L}_{k}\right\}$ and $\mathcal{H}=\left\{\mathcal{H}_{k}\right\}$, where $\mathcal{L}_{k} f(x)=f *(\phi * \sigma)_{2^{k}}(x)$ and $\mathcal{H}_{k} f(x)=f *\left[\left(\delta_{0}-\phi\right) * \sigma\right]_{2^{k}}(x)$. By (3) it suffices to bound $\lambda\left[N_{\lambda}(\mathcal{L} f)\right]^{1 / 2}$ and $\lambda\left[N_{\lambda}(\mathcal{H} f)\right]^{1 / 2}$ separately. Since the compactly supported measure $\nu:=\left(\delta_{0}-\phi\right) * \sigma$ has vanishing mean value and satisfies condition (10), we recall from [21] that the square function

$$
g(f)(x)=\left(\sum_{k \in \mathbb{Z}}\left|\mathcal{H}_{k} f(x)\right|^{2}\right)^{1 / 2}
$$

satisfies $\|g(f)\|_{p} \leq C_{p}\|f\|_{p}$, for $1<p<\infty$. Furthermore, if $\sigma$ satisfies the stronger hypothesis (11), weak-type $(1,1)$ bounds also hold. Since the pointwise estimate

$$
\lambda^{2} N_{\lambda}(\mathcal{H} f)(x) \leq 2[g(f)(x)]^{2}
$$

holds, matters are reduced to bounding $\lambda\left[N_{\lambda}(\mathcal{L} f)\right]^{1 / 2}$.

We now introduce the dyadic martingale sequence $\mathbb{E}=\left\{\mathbb{E}_{k}\right\}$ defined in (27) with respect to the dilation group $\left\{t^{P}\right\}$. As before we set $\ell=\ell(k)$ to be the integer so that $2^{\ell} \leq A^{k}<2^{\ell+1}$, and as in (3),

$$
N_{\lambda}(\mathcal{L} f)(x) \leq N_{\lambda / 2}(\mathcal{D} f)(x)+N_{\lambda / 2}(\mathbb{E} f)(x)
$$

where $\mathcal{D}=\left\{D_{k}\right\}$ is the family

$$
D_{k} f(x)=(\phi * \sigma)_{2^{\ell(k)}} * f(x)-\mathbb{E}_{k} f(x) .
$$

For $1<p<\infty$ we have the inequalities

$$
\left\|\lambda \sqrt{N_{\lambda}(\mathbb{E} f)}\right\|_{p} \leq C_{p}\|f\|_{p}
$$

moreover we have weak-type $(1,1)$ bounds. To obtain this result from (4) we fix $f \in L^{p}$ and observe that $\left|\mathbb{E}_{k} f\right| \leq C A^{-k(t r(P) / p}\|f\|_{p}$ (cf. the remark following the 
statement of Lemma 3.1). Fix $\lambda$ and choose $M$ so that $C A^{-k(t r(P) / p)}\|f\|_{p} \leq \lambda / 3$ for $k \geq M$. Let $\left\{Q_{\alpha}^{M}\right\}$ be the 'grid' of (generalized) dyadic cubes defined in (27) and, for any $\alpha$, consider the martingale $\left\{F_{N}^{M, \alpha}\right\}_{N=0}^{\infty}$ with $F_{N}^{M, \alpha}=\chi_{Q_{\alpha}^{M}} \mathbb{E}_{M-N} f$. We apply (4) and sum the estimates

$$
\left\|\lambda \sqrt{N_{\lambda}\left(F^{M, \alpha}\right)}\right\|_{L^{p}\left(Q_{\alpha}^{M}\right)}^{p} \leq \int_{Q_{\alpha}^{M}}|f(x)|^{p} d x
$$

in $\alpha$ to obtain (32); similarly one gets weak-type $(1,1)$ bounds from (5). Next, observe $\lambda^{2} N_{\lambda}(\mathcal{D} f)(x) \leq 2[\mathcal{S} f(x)]^{2}$, where

$$
\mathcal{S} f(x)=\left(\sum_{k \in \mathbb{Z}}\left|(\phi * \sigma)_{2^{\ell(k)}} * f(x)-\mathbb{E}_{k} f(x)\right|^{2}\right)^{1 / 2},
$$

and thus the proof of Theorem 1.1 reduces to bounding the square function $\mathcal{S}$. In fact, we will show that $\mathcal{S}$ is bounded on all $L^{p}\left(\mathbb{R}^{d}\right), 1<p<\infty$ and weak-type $(1,1)$; the compactly supported measure $\sigma$ no longer plays any role since $\psi:=\phi * \sigma$ is a Schwartz function whenever $\phi$ is a Schwartz function.

First we observe that the $L^{2}$ bounds for $\mathcal{S}$ follow quickly from Lemma 3.2 In fact, setting $\psi_{\ell}=(\phi * \sigma)_{2^{\ell}}$ and decomposing $f=-\sum_{m} \mathbb{D}_{m} f$ for $f \in L^{1} \cap L^{2}$, we have by (28),

$$
\begin{aligned}
\|\mathcal{S} f\|_{2} & =\left(\sum_{k \in \mathbb{Z}}\left\|\psi_{\ell(k)} * f-\mathbb{E}_{k} f\right\|_{2}^{2}\right)^{1 / 2} \\
& \leq\left(\sum_{k \in \mathbb{Z}}\left(\sum_{m \in \mathbb{Z}}\left\|\psi_{\ell(k)} * \mathbb{D}_{m} f-\mathbb{E}_{k}\left(\mathbb{D}_{m} f\right)\right\|_{2}\right)^{2}\right)^{1 / 2} \\
& \leq C\left(\sum_{k \in \mathbb{Z}}\left(\sum_{m \in \mathbb{Z}} 2^{-\delta|k-m|}\left\|\mathbb{D}_{m} f\right\|_{2}\right)^{2}\right)^{1 / 2} \\
& \leq C_{\delta}\left(\sum_{m \in \mathbb{Z}}\left\|\mathbb{D}_{m} f\right\|_{2}^{2}\right)^{1 / 2} \leq C\|f\|_{2}
\end{aligned}
$$

Next we establish weak-type $(1,1)$ bounds for $\mathcal{S}$, that is,

$$
\left|\left\{x \in \mathbb{R}^{d}: \mathcal{S} f(x)>\alpha\right\}\right| \leq \frac{C}{\alpha}\|f\|_{1} .
$$

From this, interpolation and duality give us all the $L^{p}$ bounds for $1<p<\infty$. To establish (33) we perform the Calderón-Zygmund decomposition of $f$ at height $\alpha$ using the dyadic cubes introduced in section 3. We therefore can write $f=g+b$ where $\|g\|_{\infty} \leq \alpha,\|g\|_{1} \leq\|f\|_{1}$ and $b$ is supported on a disjoint collection of dyadic cubes $\left\{Q_{\beta}^{j}\right\}_{(j, \beta) \in \Lambda}$ whose union has measure at most $C \alpha^{-1}\|f\|_{1}$. More precisely,

$$
b(x)=\sum_{(j, \beta) \in \Lambda} b_{j, \beta}(x)
$$

where $b_{j, \beta}=f-\mathbb{E}_{j} f$ on its support $Q_{\beta}^{j}$. Thus $\sum\left\|b_{j, \beta}\right\|_{1} \leq 2\|f\|_{1}$ and $\mathbb{E}_{k} b_{j, \beta}=0$ whenever $k \geq j$.

As usual the $L^{2}$ bounds for $\mathcal{S}$ allow us to handle the good function $g$ :

$$
\alpha^{2}|\{x: \mathcal{S} g(x) \geq \alpha\}| \leq C\|\mathcal{S} g\|_{2}^{2} \leq C\|g\|_{2}^{2} \leq C \alpha\|f\|_{1} .
$$


For $b$, we need only make estimates away from the union of fixed dilates, $\widetilde{Q}_{\beta}^{j}$, of $Q_{\beta}^{j}$ and then

$$
\begin{aligned}
\alpha \mid\left\{x \notin \bigcup \widetilde{Q}_{\beta}^{j}\right. & : \mathcal{S} b(x) \geq \alpha\} \mid \\
& \leq \sum_{(j, \beta) \in \Lambda} \sum_{k} \int_{\mathbb{R}^{d} \backslash \widetilde{Q}_{\beta}^{j}}\left|\psi_{\ell(k)} * b_{j, \beta}(x)-\mathbb{E}_{k} b_{j, \beta}(x)\right| d x \\
& =\sum_{(j, \beta) \in \Lambda} \sum_{k} \int_{\mathbb{R}^{d} \backslash \widetilde{Q}_{\beta}^{j}}\left|\psi_{\ell(k)} * b_{j, \beta}(x)\right| d x .
\end{aligned}
$$

The last equality holds since $\mathbb{E}_{k} b_{j, \beta}$ is supported in $Q_{\beta}^{j}$ when $k \leq j$, and as we have already noted, $\mathbb{E}_{k} b_{j, \beta}$ vanishes everywhere when $k \geq j$. For $k \leq j$ we estimate

$$
\begin{aligned}
\int_{\mathbb{R}^{d} \backslash \widehat{Q}_{\beta}^{j}} & \left|\psi_{\ell(k)} * b_{j, \beta}(x)\right| d x \\
& \leq C_{N} \int\left|b_{j, \beta}(y)\right| \int_{\left\{x: \rho\left(x-z_{\beta}^{j}\right) \geq C A^{j}\right\}} \frac{A^{-(\operatorname{tr} P) k}}{\left[A^{-k} \rho(x-y)\right]^{N}} d x d y \\
& \leq C_{N} A^{-N(j-k)}\left\|b_{j, \beta}\right\|_{1} .
\end{aligned}
$$

For $k>j$, we write

$$
\psi_{\ell(k)} * b_{j, \beta}(x)=\int\left[\psi_{\ell(k)}(x-y)-\psi_{\ell(k)}\left(x-z_{\beta}^{j}\right)\right] b_{j, \beta}(y) d y,
$$

which allows us to estimate, using (29),

$$
\begin{aligned}
\int_{\mathbb{R}^{d} \backslash \widetilde{Q}_{\beta}^{j}} \mid & \psi_{\ell(k)} * b_{j, \beta}(x) \mid d x \\
& \leq C_{N} A^{-\delta(k-j)} \int\left|b_{j, \beta}(y)\right| \int \frac{A^{-(\operatorname{tr} P) k}}{\left[1+A^{-k} \rho(x-y)\right]^{N}} d x d y \\
& \leq C_{N} A^{-\delta(k-j)}\left\|b_{j, \beta}\right\|_{1} .
\end{aligned}
$$

From (34) and (35), we obtain the desired bound

$$
\alpha\left|\left\{x \notin \bigcup \widetilde{Q}_{\beta}^{j}: \mathcal{S} b(x) \geq \alpha\right\}\right| \leq C \sum_{(j, \beta) \in \Lambda} \int\left|b_{j, \beta}(x)\right| d x \leq C\|f\|_{1},
$$

finishing the proof of the weak-type $(1,1)$ bound for $\mathcal{S}$ and therefore the proof of Theorem 1.1 .

\section{Proof of Theorems 1.2 and 1.6}

Proof of Theorem 1.2. Here we are looking at the family $\mathcal{T}=\left\{T_{k}\right\}$ of truncated singular integral operators $T_{k} f(x)=\sum_{j \geq k} \nu_{2^{j}} * f(x)$ where $\nu$ is a compactly supported finite Borel measure with cancellation $\widehat{\nu}(0)=0$, and $\nu_{2^{j}}$ is the $2^{j P}$ - dilate of $\nu$. Our goal is to bound the $\lambda$-jump operator $f \rightarrow N_{\lambda}(\mathcal{T} f)$ under the regularity conditions (10) or (11) for $\nu$.

To establish $L^{p}, 1<p<\infty$, bounds under the weak regularity condition (10), we begin in the same way as in 21] where the corresponding maximal truncation 
operator, $T_{*} f(x)=\sup _{k}\left|T_{k} f(x)\right|$ was treated. Let $\phi$ be a smooth function with compact support such that $\int \phi=1$. Writing $T f=\sum_{j} \nu_{2^{j}} * f$, we decompose

$$
\begin{aligned}
T_{k} f & =\phi_{2^{k}} *\left(T f-\sum_{j<k} \nu_{2^{j}} * f\right)+\left(\delta_{0}-\phi_{2^{k}}\right) * \sum_{j \geq k} \nu_{2^{j}} * f \\
& =\phi_{2^{k}} * T f-\left[\phi * \sum_{\ell \leq 0} \nu_{2^{\ell}}\right]_{2^{k}} * f+\sum_{s \geq 0}\left(\delta_{0}-\phi_{2^{k}}\right) * \nu_{2^{k+s}} * f,
\end{aligned}
$$

which divides $\mathcal{T}$ into three families

$$
\begin{aligned}
& \mathcal{T}^{1} f=\left\{\phi_{2^{k}} * T f\right\}, \\
& \mathcal{T}^{2} f=\left\{\left[\phi * \sum_{\ell \leq 0} \nu_{2^{\ell}}\right]_{2^{k}} * f\right\}, \\
& \mathcal{T}^{3} f=\left\{\left(\delta_{0}-\phi_{2^{k}}\right) * \sum_{s \geq 0} \nu_{2^{k+s}} * f\right\}
\end{aligned}
$$

and as in (3) it suffices to bound $\lambda\left[N_{\lambda}\left(\mathcal{T}^{i} f\right)\right]^{1 / 2}$, for each $i=1,2,3$ separately.

For $\mathcal{T}^{3}$ we estimate

$$
\lambda\left[N_{\lambda}\left(\mathcal{T}^{3} f\right)(x)\right]^{1 / 2} \leq C \sum_{s \geq 0} G_{s} f(x),
$$

where $G_{s} f(x)=\left(\sum_{k \in \mathbb{Z}}\left|\left[\left(\delta_{0}-\phi\right) * \nu_{2^{s}}\right]_{2^{k}} * f(x)\right|^{2}\right)^{1 / 2}$. Since for each $s>0$, the measure $\left(\delta_{0}-\phi\right) * \nu_{2^{s}}$ is supported in a fixed compact set with vanishing mean value and also satisfying (10) uniformly in $s$, we see that $G_{s}$ is bounded on all $L^{p}, 1<p<\infty$, uniformly in $s$. Furthermore, using Plancherel's theorem and the estimate

$$
\sum_{k \in \mathbb{Z}}\left[\left|1-\widehat{\phi}\left(2^{k P^{*}} \xi\right) \| \widehat{\nu}\left(2^{(k+s) P^{*}} \xi\right)\right|\right]^{2} \leq C 2^{-\delta s}
$$

for some $\delta>0$ which follows from (10), we see that $\left\|G_{s} f\right\|_{2} \leq C 2^{-\delta s}\|f\|_{2}$. Interpolation and duality imply that the uniform $L^{p}$ bounds for $G_{s}$ extend to $\left\|G_{s} f\right\|_{p} \leq C_{p} 2^{-\delta_{p} s}\|f\|_{p}, 1<p<\infty$, for some $\delta_{p}>0$, giving us the desired $L^{p}$ bounds for $\lambda\left[N_{\lambda}\left(\mathcal{T}^{3} f\right)\right]^{1 / 2}$.

The argument for $\mathcal{T}^{2}$ is similar but easier since the compactly support measure $\phi * \sum_{\ell \leq 0} \nu_{2^{\ell}}$ has vanishing mean value with rapidly decaying Fourier transform. Hence the pointwise estimate

$$
\lambda\left[N_{\lambda}\left(\mathcal{T}^{2} f\right)(x)\right]^{1 / 2} \leq C\left(\sum_{k \in \mathbb{Z}}\left|\left[\phi * \sum_{\ell \leq 0} \nu_{2^{\ell}}\right]_{2^{k}} * f(x)\right|^{2}\right)^{1 / 2}
$$

establishes the $L^{p}$ bounds for $\lambda\left[N_{\lambda}\left(\mathcal{T}^{2} f\right)\right]^{1 / 2}$. Finally we can apply Theorem 1.1 to the family given by $\mathfrak{A}_{k} f=\phi_{2^{k}} * f$ to obtain

$$
\left\|\lambda\left[N_{\lambda}\left(\mathcal{T}^{1} f\right)\right]^{1 / 2}\right\|_{p} \leq C_{p}\|T f\|_{p} \leq C_{p}^{\prime}\|f\|_{p}
$$

for $1<p<\infty$.

Although it is true that under the stronger regularity condition for $\nu$ (that is, $d \nu(x)=\Psi(x) d x$, where $\Psi$ is an $L^{1}$ function satisfying the $L^{1}$ smoothness condition (11)) one can establish the weak-type $(1,1)$ bounds

$$
\alpha\left|\left\{x: G_{s} f(x)>\alpha\right\}\right| \leq C 2^{-\epsilon s}\|f\|_{1}
$$

for some $\epsilon>0$, and similarly for the square function appearing in (36), we are still left with the family $\left\{\phi_{2^{k}} * T\right\}$ and $T$ is not bounded on $L^{1}$ in general. Instead 
we take the direct approach used in establishing (33) and perform the CalderónZygmund decomposition of $f$ at height $\alpha$, producing a disjoint family of dyadic cubes $\{Q\}$ with total measure $\sum|Q| \leq C / \alpha\|f\|_{1}$ and allowing us to write $f=g+b$ with $\|g\|_{\infty} \leq C \alpha,\|g\|_{1} \leq C\|f\|_{1}$ and $b=\sum_{Q} b_{Q}$, where each $b_{Q}$ is supported in $Q$ and has mean value zero such that $\sum\left\|b_{Q}\right\|_{1} \leq C\|f\|_{1}$. Since we already know that the $L^{2}$ norm of $\lambda\left[N_{\lambda}(\mathcal{T} g)\right]^{1 / 2}$ is uniformly controlled by the $L^{2}$ norm of $g$, matters are reduced in the usual way to estimating $\lambda\left[N_{\lambda}(\mathcal{T} b)\right]^{1 / 2}$ away from $\bigcup \widetilde{Q}$ where $\widetilde{Q}$ is a fixed large dilate of $Q$. Using the pointwise estimate

$$
\lambda\left[N_{\lambda}(\mathcal{T} b)(x)\right]^{1 / 2} \leq \sum_{k \in \mathbb{Z}}\left|T_{k} b(x)-T_{k+1} b(x)\right| \leq \sum_{k \in \mathbb{Z}}\left|\Psi_{2^{k}} * b(x)\right|
$$

we see that

$$
\alpha\left|\left\{x \notin \bigcup \widetilde{Q}: \lambda\left[N_{\lambda}(\mathcal{T} b)(x)\right]^{1 / 2}>\alpha\right\}\right| \leq \sum_{Q} \sum_{k} \int_{x \notin \widetilde{Q}}\left|\Psi_{2^{k}} * b_{Q}(x)\right| d x .
$$

Since $\Psi$ has compact support, $\Psi_{2^{k}} * b_{Q}$ vanishes off $\widetilde{Q}$ when $k<k(Q)$ (here $2^{k(Q)}$ is roughly the diameter of $Q$ described in part iv) of Lemma 3.1 and we are taking a large but fixed dilate in the definition of $\widetilde{Q}$ ). Thus, using the vanishing mean value of $b_{Q}$, the right side of the above inequality is dominated by

$$
\sum_{Q} \sum_{k \geq k(Q)} \int\left|b_{Q}(y)\right| \int_{x \notin \widetilde{Q}}\left|\Psi_{2^{k}}(x-y)-\Psi_{2^{k}}\left(x-y_{Q}\right)\right| d x d y
$$

where $y_{Q}$ denotes the 'center' of $Q$ as described in Lemma 3.1 This in turn, using condition (11), is

$$
\leq C \sum_{Q} \sum_{k \geq k(Q)} 2^{-\delta(k-k(Q))}\left\|b_{Q}\right\|_{1} \leq C^{\prime}\|f\|_{1},
$$

establishing the uniform weak-type $(1,1)$ bound for $f \rightarrow \lambda\left[N_{\lambda}(\mathcal{T} f)\right]^{1 / 2}$ and therefore finishing the proof of Theorem 1.2 .

Proof of Theorem 1.6. Define the measure $\nu$ by

$$
\langle\nu, f\rangle=\int_{1 \leq|y| \leq 2} \Omega(y /|y|)|y|^{-d} f(y) d y .
$$

It is well known (see, e.g., 21] ) that under the assumption $\Omega \in L^{r}\left(S^{d-1}\right)$ the Fourier transform

$$
\widehat{\nu}(\xi)=\int_{S^{d-1}} \Omega(\theta) \int_{1}^{2} e^{-i\langle r \theta, \xi\rangle} r^{-1} d r d \theta
$$

decays; it satisfies

$$
|\widehat{\nu}(\xi)| \leq C\|\Omega\|_{L^{r}\left(S^{d-1}\right)} \min \left\{|\xi|,|\xi|^{-\frac{r-1}{2 r-1}}\right\} .
$$

The bound for small $\xi$ follows by the assumed cancellation of $\Omega$. To see the bound for $|\xi| \geq 1$ we split the $\theta$-integral in (37) and consider first the region for $|\langle\theta, \xi /|\xi|\rangle| \leq a$ for small $a$; here we apply Hölders inequality. For $\theta$ in the complementary region integrate by parts in $r$ once to gain $(|\xi| a)^{-1}$, then optimize the two bounds with the choice $a=|\xi|^{-r /(2 r-1)}$.

Now the $L^{p}$ inequalities for $N_{\lambda}^{\text {dyad }}(\mathcal{T} f)$ follow from Theorem 1.2. The full claim follows by combining Lemma 1.3 and a result on the short-variation operator. The relevant $L^{p}$ bound for the short-variation $\left(S_{2^{-}}\right)$bound has been proved for 
the Hilbert transform in [9], and the method of rotation argument used for $V^{q}(\mathcal{T})$ in [10] applies also to the short variation operator. Thus one gets the $L^{p}$ bounds for $S_{2}(\mathcal{T} f)$ under the weaker assumption $\Omega \in L \log L\left(S^{d-1}\right)$ (and in fact $\Omega \in L^{1}\left(S^{d-1}\right)$ for odd $\Omega$ ).

\section{Prelude to short variations}

For a general family of linear operators $\mathcal{A}=\left\{A_{t}\right\}$ indexed say by positive $t>0$, we have divided the $\lambda$-jumps $N_{\lambda}(\mathcal{A} f)$ into short and long jumps by using inequality (16). We have already treated the dyadic $\lambda$-jumps $N_{\lambda}^{\text {dyad }}$ for a large class of convolution operators on $\mathbb{R}^{d}$ in Theorems 1.1 and 1.2. and so we turn our attention to the short $\rho$-variation operators $S_{\rho} \circ \mathcal{A}$ defined in (15), (14) (we shall mostly assume $\rho=2)$.

As mentioned in the introduction known techniques from harmonic analysis can be used to treat $S$. In the early 1970s, Stein introduced square function techniques to understand singular maximal functions such as those arising from spherical means (17) or parabolic averages (21); these techniques amount to essentially using a version of a Sobolev-imbedding theorem in the $t$ variable (see also [11, 12, 19], [39]). In fact, viewing the value of the maximal function $M f(x)=\sup _{t>0}\left|A_{t} f(x)\right|$ as an $L^{\infty}$ norm on $\mathbb{R}^{+},\|\mathcal{A} f(x)\|_{L^{\infty}\left(\mathbb{R}^{+}\right)}$one can use the easy bound

$$
\|a\|_{L^{\infty}\left(\mathbb{R}^{+}\right)} \leq\|a\|_{L^{\rho}\left(\mathbb{R}^{+}\right)}^{1 / \rho^{\prime}}\left\|a^{\prime}\right\|_{L^{\rho}\left(\mathbb{R}^{+}\right)}^{1 / \rho}+a\left(t_{0}\right),
$$

which holds by the fundamental theorem of calculus for any $a$. Using this for $\rho=2$ one brings $L^{2}$ methods into play in a decisive way to study $M f$. However (38) remains valid if we replace the $L^{\infty}$ norm of $a$ with the larger $v_{\rho}$ norm (see (11)). This is most easily seen via the Besov space embedding $\dot{B}_{\rho, 1}^{1 / \rho} \subset v_{\rho}$ established by Bergh and Peetre [2,

$$
\|a\|_{v_{\rho}} \leq C \sum_{j \in \mathbb{Z}} 2^{j / \rho}\left\|\phi_{j} * a\right\|_{\rho} \leq C\|a\|_{\rho}^{1 / \rho^{\prime}}\left\|a^{\prime}\right\|_{\rho}^{1 / \rho}
$$

where $\phi_{j}(x)=2^{j} \phi\left(2^{j} x\right)$ and $\phi$ is a $\operatorname{Schwartz}$ function such that $\operatorname{supp}(\widehat{\phi}) \subset\{1 / 2 \leq$ $|\xi| \leq 2\}$ and $\sum_{j \in \mathbb{Z}} \widehat{\phi}\left(2^{-j} \xi\right)=1$ for $\xi \neq 0$. The middle expression in (39) defines a norm, modulo constants, for $\dot{B}_{\rho, 1}^{1 / \rho}$.

The second estimate in (39) is a standard interpolation inequality while the first follows from the estimate $\left\|\phi_{j} * a\right\|_{v_{\rho}} \leq C 2^{j / \rho}\left\|\phi_{j} * a\right\|_{\rho}$, which can be proved by using the Plancherel-Polya inequality.

Although our main interest is to understand thoroughly the short 2-variation operator in particular cases, we illustrate here how one can use (39) to extend some results of Rubio de Francia [39] in the general context of convolution operators $A_{t} f(x)=f * \sigma_{t}(x)$ on $\mathbb{R}^{d}$ where $\sigma_{t}$ is the $t^{P}$-dilate of a compactly supported finite Borel measure $\sigma$ satisfying the weak regularity condition (10). The proof of the following lemma is a straightforward adaptation of the arguments in [39], using (39) at the appropriate places. We shall only give the main points of the proof.

Lemma 6.1. Let $\mathcal{A}=\left\{A_{t}\right\}$ where $A_{t} f=f * \sigma_{t}$ is described above. Suppose that $\sigma$ satisfies (10) for some $b>1 / 2$, Then the a priori estimate

$$
\left\|S_{2}(\mathcal{A} f)\right\|_{L^{p}\left(\mathbb{R}^{d}\right)} \leq C_{p}\|f\|_{L^{p}\left(\mathbb{R}^{d}\right)}
$$

holds for $\min (2 d /(d+2 b-1),(2 b+1) /(2 b))<p<\max (2 d /(d-2 b+1), 2 b+1)$. 
Remarks. (i) It is understood that the lower bound $p_{1}=\min (2 d /(d+2 b-1)$, $(2 b+1) /(2 b))$ is equal to 1 and the upper bound $p_{2}=\max (2 d /(d-2 b+1), 2 b+1)$ is equal to $\infty$ when $b \geq(d+1) / 2$. If $\sigma$ is absolutely continuous with $L^{1}$ density the characteristic function of the unit ball, then $b=(d+1) / 2$.

(ii) As a consequence of (16) and Theorem 1.1 we obtain uniform $L^{p}$ bounds for $\lambda\left[N_{\lambda}(\mathcal{A} f)\right]^{1 / 2}$ in the same range of $p$. Therefore Lemma 2.1 implies that these $L^{p}$ bounds also hold for $V_{q}(\mathcal{A} f)$ whenever $q>2$ since the open range of $p$ always contains $p=2$. Interpolation with the trivial $L^{\infty}$ estimate for the corresponding maximal operator shows that for $p \geq p_{2}$,

$$
\left\|V_{q}(\mathcal{A} f)\right\|_{p} \leq C_{p, q}\|f\|_{p}
$$

holds when $q>2 p / p_{2}$.

(iii) Applying Lemma 6.1 to the family of spherical means (17) gives strong $q$-variational estimates for $q>2$ only in the range $d /(d-1)<p \leq d$ and then only for $d \geq 3$. The proof of Theorem 1.4 also uses square function estimates but these estimates lie much deeper than those employed to prove Lemma 6.1. Also the result stated in Theorem 1.5 is not covered by Lemma 6.1 since in this case, $b=1 / 2$ and in fact we obtain $L^{p}$ bounds for the full range $1<p<\infty$. The proof of Theorem 1.5] exploits the added feature that the parabolic dilations flow along (not transversal to) the support of $\sigma$.

We now sketch the proof of Lemma 6.1, using the notation in [39]. We begin by decomposing $\sigma=\sum_{k \geq 0} \sigma * \psi_{k}$ where $\psi_{0}$ is a Schwartz function such that $\widehat{\psi}_{0}(\xi)=1$ if $|\xi| \leq 1$ and $\widehat{\psi}_{0}(\xi)=0$ if $|\xi| \geq 2$ and $\widehat{\psi}_{k}(\xi)=\widehat{\psi}_{0}\left(2^{-k} \xi\right)-\widehat{\psi}_{0}\left(2^{-(k-1)} \xi\right)$. Therefore

$$
S_{2}(\mathcal{A} f)(x) \leq \sum_{k \geq 0} S_{2}\left(\mathcal{A}^{k} f\right)(x)
$$

where $\mathcal{A}^{k}=\left\{A_{t}^{k}\right\}$ and $A_{t}^{k} f=f *\left(\sigma * \psi_{k}\right)_{t}$. Using (39) we see that

$$
\begin{aligned}
{\left[S_{2}\left(\mathcal{A}^{k} f\right)(x)\right]^{2} } & \leq C \sum_{j}\left(\int_{2^{j}}^{2^{j+1}}\left|A_{t}^{k} f(x)\right|^{2} \frac{d t}{t}\right)^{1 / 2}\left(\int_{2^{j}}^{2^{j+1}}\left|\widetilde{A}_{t}^{k} f(x)\right|^{2} \frac{d t}{t}\right)^{1 / 2} \\
& \leq C G_{k} f(x) \widetilde{G}_{k} f(x)
\end{aligned}
$$

where $G_{k} f(x)^{2}=\int_{0}^{\infty}\left|A_{t}^{k} f(x)\right|^{2} d t / t$ is a square function associated to the multiplier $m_{k}=\widehat{\psi}_{k} \widehat{\sigma}$ and $\widetilde{G}_{k}$ is defined in the same way but with respect to the multiplier $\widetilde{m}_{k}=\left\langle P^{*} \xi, \nabla m_{k}(\xi)\right\rangle$. Since $\left\|m_{k}\right\|_{\infty} \leq C 2^{-k b},\left\|\widetilde{m}_{k}\right\|_{\infty} \leq C 2^{k(1-b)}$ and both $m_{k}, \widetilde{m}_{k}$ are supported where $|\xi| \sim 2^{k}$, we have by Plancherel's theorem,

$$
\left\|S_{2}\left(\mathcal{A}^{k} f\right)\right\|_{2}^{2} \leq C\left\|G_{k} f\right\|_{2}\left\|\widetilde{G}_{k} f\right\|_{2} \leq C 2^{-(2 b-1) k}\|f\|_{2}^{2},
$$

which establishes the $L^{2}$ bound for $S_{2}(\mathcal{A} f)$ since $b>1 / 2$.

Furthermore it was shown in [39] that for $\alpha>-b+d / 2$ we have the estimates

$$
\left\|G_{k} f\right\|_{1} \leq C 2^{k \alpha}\|f\|_{H^{1}} \quad \text { and } \quad\left\|G_{k} f\right\|_{B M O} \leq C 2^{k \alpha}\|f\|_{\infty}
$$

and

$$
\left\|\widetilde{G}_{k} f\right\|_{1} \leq C 2^{k(1+\alpha)}\|f\|_{H^{1}} \quad \text { and } \quad\left\|\widetilde{G}_{k} f\right\|_{B M O} \leq C 2^{k(1+\alpha)}\|f\|_{\infty} .
$$

Here $H^{1}$ and $B M O$ are defined with respect to the dilation group $\left\{t^{P}\right\}$. Defining

$$
\mathcal{N}_{k} f(x)=2^{k / 2} G_{k} f(x)+2^{-k / 2} \widetilde{G}_{k} f(x),
$$


we can then estimate

$$
\begin{aligned}
& \left\|\mathcal{N}^{k} f\right\|_{1} \leq C 2^{(1 / 2+s-b) k}\|f\|_{H^{1}}, \\
& \left\|\mathcal{N}^{k} f\right\|_{B M O} \leq C 2^{(1 / 2+\alpha) k}\|f\|_{\infty},
\end{aligned} \quad \alpha>\frac{d}{2}-b .
$$

Since (40) implies

$$
S_{2}\left(\mathcal{A}^{k} f\right)(x) \leq C\left[G_{k} f(x) \widetilde{G}_{k} f(x)\right]^{1 / 2} \leq C \mathcal{N}_{k} f(x),
$$

we can interpolate with the $L^{2}$ estimates in (41) to show that $S_{2}(\mathcal{A} f)$ is bounded on $L^{p}$ for $|1 / p-1 / 2|<b / d-1 /(2 d)$, which establishes the lemma except for the improved range when $b<(d-1) / 2$.

To achieve this improvement we use vector-valued singular integral theory for the convolution operators $\left\{T_{k, t} f=f * K_{k, t}\right\}_{t>0}$ where we view the kernel $K_{k}(x)=$ $\left\{K_{k, t}(x)=\left(\sigma * \psi_{k}\right)_{t}(x)\right\}_{t>0}$ as having values in the Banach space

$$
B=\left\{a(t):\|a\|_{B}:=\left(\sum_{j}\|a\|_{V_{2}\left[2^{j}, 2^{j+1}\right]}^{2}\right)^{1 / 2}<\infty\right\}
$$

used in the definition of the short 2-variation operator $S(\mathcal{A} f)$. Then we have the $L^{p}$ estimates

$$
\left\|S_{2}\left(\mathcal{A}^{k} f\right)\right\|_{p} \leq C_{p}\left[M_{k}+N_{k}\right]\|f\|_{p}
$$

for $1<p<\infty$, where $M_{k}=2^{-(b-1 / 2) k}$ is the already established $L^{2}$ bound (41) and $N_{k}$ is any upper bound for

$$
\sup _{y \in \mathbb{R}^{d}} \int_{\rho(x) \geq C_{0} \rho(y)}\left\|K_{k}(x-y)-K_{k}(x)\right\|_{B} d x
$$

where $\rho$ is homogeneous with respect to $\left\{t^{P}\right\}$ and $C_{0}>1$ is some fixed large constant; see, e.g., 22. To estimate this integral we simply use the fact that the 2 -variation norm is dominated by the 1 -variation norm, which gives us the trivial estimate

$$
\|a\|_{B} \leq \int_{0}^{\infty}\left|a^{\prime}(t)\right| d t
$$

and therefore

$$
\begin{aligned}
\int_{\rho(x) \geq C_{0} \rho(y)}\left\|K_{k}(x-y)-K_{k}(x)\right\|_{B} d x & \\
& \leq \int_{0}^{\infty} \int_{\rho(x) \geq C_{0} \rho(y)}\left|\widetilde{K}_{k, t}(x-y)-\widetilde{K}_{k, t}(x)\right| d x \frac{d t}{t},
\end{aligned}
$$

where $\widetilde{K}_{k, t}$ is the convolution kernel for $\widetilde{T}_{k, t}$. In [39] it was shown that

$$
\int_{0}^{\infty} \int_{\rho(x) \geq C_{0} \rho(y)}\left|\widetilde{K}_{k, t}(x-y)-\widetilde{K}_{k, t}(x)\right| d x \frac{d t}{t} \leq C\|\sigma\| k 2^{k}
$$

uniformly in $y$ and so $N_{k}=k 2^{k}$ is an upper bound for the integral in (45), which gives us $\left\|S_{2}\left(\mathcal{A}^{k} f\right)\right\|_{p} \leq C_{p} k 2^{k}\|f\|_{p}$ for all $1<p<\infty$. Interpolating these estimates with the $L^{2}$ estimates in (41) shows that $S_{2}(\mathcal{A} f)$ is bounded on $L^{p}$ in the range $(2 b+1) /(2 b)<p<2 b+1$, completing the main points in the proof of Lemma 6.1 . 


\section{Proofs of Theorems 1.5 and 1.7}

Proof of Theorem 1.5. Here we are considering the family of averaging operators $\mathcal{A}=\left\{A_{t}\right\}$ on $\mathbb{R}^{2}$ given by $A_{t} f=f * \sigma_{t}$, where the measure $\sigma$ is defined on a test function $\phi$ by $\langle\sigma, \phi\rangle=\int_{0}^{1} \phi\left(s, s^{a}\right) d s$ and $\sigma_{t}$ is the nonisotropic dilate of $\sigma$ given in (9) with $t^{P} x=\left(t x_{1}, t^{a} x_{2}\right)$ (i.e., $P$ is the diagonal matrix with eigenvalues 1 and $a)$. In what follows we assume that $a>0, a \neq 1$, since in the case $a=1$ we can apply results for the standard one-dimensional averages. We shall establish $L^{p}\left(\mathbb{R}^{2}\right), 1<p<\infty$, bounds for the corresponding short 2-variation operator,

$$
\left\|S_{2}(\mathcal{A} f)\right\|_{p} \leq C_{p}\|f\|_{p}
$$

from which uniform $L^{p}, 1<p<\infty$, bounds for $\lambda\left[N_{\lambda}(\mathcal{A} f)\right]^{1 / 2}$ follow from Theorem 1.1 and (16). Then Lemma 2.1 implies the desired result for the strong $q$-variations $V_{q}(\mathcal{A} f)$ in Theorem 1.5 .

The rough cutoff in the definition of $\sigma$ causes some difficulties. If, instead, we consider the family of convolution operators defined by $f * \mu_{t}$, where the measure $\mu$ on $\mathbb{R}^{2}$ is defined by

$$
\langle\mu, f\rangle=\int f\left(s, s^{a}\right) \psi(s) d s
$$

and $\psi \in C_{0}^{\infty}((1 / 2,4))$, then the methods in the proof of Lemma 6.1 apply in a straightforward fashion even though the optimal decay rate $|\widehat{\mu}(\xi)| \leq|\xi|^{-1 / 2}$ prevents us from applying the lemma directly. Since the support of $\mu$ lies along an orbit of $\left\{t^{P}\right\}$ we can improve the estimate $\left|t(d / d t) \widehat{\mu_{t}}(\xi)\right| \leq\left|t^{P^{*}} \xi\right|^{1 / 2}$ used in the proof of Lemma 6.1 to $\left|t(d / d t) \widehat{\mu_{t}}(\xi)\right| \leq\left|t^{P^{*}} \xi\right|^{-1 / 2}$ (this can be verified by a direct computation, see (49) below; however without the smooth cutoff $\psi$, one obtains only a uniform estimate with no decay in $\left.\left|t^{P^{*}} \xi\right|\right)$. This allows us to improve (41) to $\left\|S_{2}\left(\mathcal{A}^{k} f\right)\right\|_{2} \leq C 2^{-k / 2}\|f\|_{2}$ and (46) to

$$
\int_{0}^{\infty} \int_{\rho(x) \geq C_{0} \rho(y)}\left|\widetilde{K}_{k, t}(x-y)-\widetilde{K}_{k, t}(x)\right| d x \frac{d t}{t} \leq C\|\mu\| k .
$$

Interpolation gives us $L^{p}$ estimates for all $1<p<\infty$.

To overcome the difficulty of the rough cutoff at $s=0$ and $s=1$ in the definition of $\sigma$, we introduce a partition of unity by writing $\sigma=\sigma^{0}+\sigma^{1}=\sum_{m>0} \sigma_{m}^{0}+\sum_{n>0} \sigma_{n}^{1}$ with

$$
\begin{aligned}
\left\langle\sigma_{n}^{0}, f\right\rangle & =\int f\left(s, s^{a}\right) \widetilde{\chi}(s) \eta\left(2^{n} s\right) d s, \\
\left\langle\sigma_{m}^{1}, f\right\rangle & =\int f\left(s, s^{a}\right) \chi(s) \eta\left(2^{m}(1-s)\right) d s,
\end{aligned}
$$

where $\chi, \widetilde{\chi}$ are smooth and $\eta$ is some smooth function supported in $(1 / 2,2)$. As in the proof of Lemma 6.1] we introduce a Littlewood-Paley decomposition and form families $\mathcal{A}^{k, m, 1}, \mathcal{A}^{k, n, 0}$, where $A_{t}^{k, m, 1} f=f *\left(\psi_{k} * \sigma_{m}^{1}\right)_{t}$ and $A_{t}^{k, n, 0} f=f *\left(\psi_{k} * \sigma_{n}^{0}\right)_{t}$, and we need to be able to sum the estimates for the corresponding short 2-variation operators $S_{2} \circ \mathcal{A}^{k, m, 1}$ and $S_{2} \circ \mathcal{A}^{k, n, 0}$ in $(k, m)$ and $(k, n)$, respectively. Since

$$
t \frac{d}{d t} e^{i\left(\xi_{1} t s+\xi_{2} t^{a} s^{a}\right)}=s \frac{d}{d s} e^{i\left(\xi_{1} t s+\xi_{2} t^{a} s^{a}\right)}
$$


we obtain by an integration by parts,

$$
\begin{aligned}
t(d / d t)\left[\widehat{\sigma_{m}^{1}}\left(t^{P^{*}} \xi\right)\right] & =t \frac{d}{d t} \int e^{i\left(\xi_{1} t s+\xi_{2} t^{a} s^{a}\right)} \chi(s) \eta\left(2^{m}(1-s)\right) d s \\
& =-\int e^{i\left(\xi_{1} t s+\xi_{2} t^{a} s^{a}\right)} \frac{d}{d s}\left[s \chi(s) \eta\left(2^{m}(1-s)\right)\right] d s
\end{aligned}
$$

and from van der Corput's lemma we get

$$
\begin{aligned}
\left|\widehat{\sigma_{m}^{1}}\left(t^{P} \xi\right)\right| & \leq C \min \left\{2^{-m},\left|t^{P^{*}} \xi\right|^{-1 / 2}\right\}, \\
\left|t \frac{d}{d t}\left[\widehat{\sigma_{m}^{1}}\left(t^{P} \xi\right)\right]\right| & \leq C \min \left\{1,2^{m}\left|t^{P^{*}} \xi\right|^{-1 / 2}\right\} .
\end{aligned}
$$

Therefore, arguing as in (40) and (41), we get

$$
\begin{aligned}
\left\|S_{2}\left(\mathcal{A}^{k, m, 1} f\right)\right\|_{2} & \leq C \min \left\{2^{-m / 2}, 2^{m / 2} 2^{-k / 4}\right\}\|f\|_{2} \\
& \leq C^{\prime} 2^{-k / 10} 2^{-m / 5}\|f\|_{2} .
\end{aligned}
$$

Similarly for $\sigma_{n}^{0}$ we have

$$
t \frac{d}{d t}\left[\widehat{\sigma_{n}^{0}}\left(t^{P^{*}} \xi\right)\right]=\int e^{i\left(\xi_{1} t s+\xi_{2} t^{a} s^{a}\right)} \frac{d}{d s}\left[s \widetilde{\chi}(s) \eta\left(2^{n} s\right)\right] d s,
$$

and we get the estimate

$$
\left|\widehat{\sigma}_{n}^{0}\left(t^{P^{*}} \xi\right)\right|+\left|\frac{d}{d t}\left[\widehat{\sigma_{n}^{0}}\left(t^{P^{*}} \xi\right)\right]\right| \leq C(a) \min \left\{2^{-n}, 2^{(2-a) n / 2}\left|t^{P^{*}} \xi\right|^{-1 / 2}\right\} .
$$

Thus also

$$
\begin{aligned}
\left\|S_{2}\left(\mathcal{A}^{k, n, 0} f\right)\right\|_{2} & \leq C(a) \min \left\{2^{-n}, 2^{(2-a) n / 2} 2^{-k / 2}\right\}\|f\|_{2} \\
& \leq C^{\prime}(a) 2^{-(k+a n) / 4}\|f\|_{2},
\end{aligned}
$$

where $C^{\prime}(a)<\infty$ for $a \neq 1$.

Using (49), one can argue as in (48) to estimate

$$
\begin{gathered}
\int_{0}^{\infty} \int_{\rho(x) \geq C_{0} \rho(y)}\left|\widetilde{K}_{k, m, t}^{1}(x-y)-\widetilde{K}_{k, m, t}^{1}(x)\right| d x \frac{d t}{t} \leq C\left\|\sigma_{m}\right\| 2^{m} k \leq C k, \\
\int_{0}^{\infty} \int_{\rho(x) \geq C_{0} \rho(y)}\left|\widetilde{K}_{k, n, t}^{0}(x-y)-\widetilde{K}_{k, n, t}^{0}(x)\right| d x \frac{d t}{t} \leq C\left\|\sigma_{n}\right\| 2^{n} k \leq C k,
\end{gathered}
$$

where $\widetilde{K}_{k, m, t}^{1}=t \frac{d}{d t}\left[\left(\psi_{k} * \sigma_{m}^{0}\right)_{t}\right], \widetilde{K}_{k, n, t}^{0}=t \frac{d}{d t}\left[\left(\psi_{k} * \sigma_{n}^{0}\right)_{t}\right]$. This gives us $L^{p}$ estimates

$$
\left\|S_{2}\left(\mathcal{A}^{k, m, 1} f\right)\right\|_{p}+\left\|S_{2}\left(\mathcal{A}^{k, n, 0} f\right)\right\|_{p} \leq C_{p} k\|f\|_{p}
$$

for all $1<p<\infty$ and interpolation with (50), (51) implies for $1<p<\infty$,

$$
\begin{gathered}
\left\|S_{2}\left(\mathcal{A}^{k, m, 1} f\right)\right\|_{p} \leq C_{p} 2^{-\delta_{p} k} 2^{-\delta_{p}^{\prime} m}\|f\|_{p}, \\
\left\|S_{2}\left(\mathcal{A}^{k, n, 0} f\right)\right\|_{p} \leq C_{p} 2^{-\delta_{p} k} 2^{-\delta_{p}^{\prime} n}\|f\|_{p}
\end{gathered}
$$

for some $\delta_{p}, \delta_{p}^{\prime}>0$. Summing in $k$ and $m, n \geq 0$ establishes (47), completing the proof of Theorem 1.5 .

Proof of Theorem 1.7. Again we may assume $a \neq 1$ since for $a=1$ one can apply the one dimensional result for the Hilbert transform. Again, by Theorem 1.2 we only have to show that

$$
\left\|S_{2}(\mathcal{T} f)\right\|_{p}=\left\|\left(\sum_{j \in \mathbb{Z}}\left|V_{2, j}(\mathcal{T} f)\right|^{2}\right)^{1 / 2}\right\|_{p} \leq C\|f\|_{p} .
$$


Now let $s \in[1,2]$ and define $\nu_{0, s}$ to be the measure given by

$$
\left\langle\nu_{0, s}, f\right\rangle=\int_{s \leq|u| \leq 2} f\left(u, u^{a}\right) \frac{d u}{u} .
$$

Define the dilate $\nu_{j, s}$ by $\left\langle\nu_{j, s}, f\right\rangle=\left\langle\nu_{0, s}, f\left(2^{j P} \cdot\right)\right\rangle$. Finally for $k \in \mathbb{Z}$, let $\Pi_{j, k} f$ be the rescaled Littlewood-Paley operator defined by

$$
\widehat{\Pi_{j, k} f}(\xi)=\varphi\left(\left|2^{j P^{*}} 2^{-k} \xi\right|\right) \widehat{f}(\xi)
$$

where $\sum_{k \in \mathbb{Z}} \varphi\left(s 2^{-k}\right) \equiv 1$ for all $s>0$. We observe that $V_{2, j}(\mathcal{T} f)(x)$ is just the $V_{2}$ variation of the family $\left\{\nu_{j, s} * f\right\}_{s \in[1,2]}$, and it suffices to establish for $1<p<\infty$ the estimates

$$
\begin{aligned}
& \left\|\left(\sum_{j \in \mathbb{Z}}\left|\nu_{j, s} * \Pi_{j, k} f\right|^{2}\right)^{1 / 2}\right\|_{p} \leq C_{p} 2^{-|k| \epsilon(p)}\|f\|_{p}, \\
& \left\|\left(\sum_{j \in \mathbb{Z}}\left|\frac{d}{d s} \nu_{j, s} * \Pi_{j, k} f\right|^{2}\right)^{1 / 2}\right\|_{p} \leq C_{p}\|f\|_{p},
\end{aligned}
$$

uniformly in $s \in[1,2]$. The proof of these estimates is very much analogous to the above estimates for the averaging operators, except we get decay also as $k \rightarrow-\infty$ in view of the cancellation of the measures $\nu_{0, s}$. We omit the details.

Remark. A similar argument also applies for curves $t \mapsto \gamma(t)$ which are homogeneous with respect to nonisotropic dilations, with a nonsymmetric $P$, e.g., with

$$
P=\left(\begin{array}{ll}
1 & 1 \\
0 & 1
\end{array}\right), \quad t^{P}=\left(\begin{array}{cc}
t & t \log t \\
0 & t
\end{array}\right) .
$$

We choose $u=(1,1)^{t}$ and $\gamma(t)=t^{P} u$ and define the maximal and singular integrals analogously to (21), (25). One still obtains the conclusions of Theorem 1.5 and Theorem 1.7, the proofs above apply with only small changes.

\section{Proof of Theorem 1.4}

As remarked earlier, Lemma 6.1 does not cover the full $L^{p}$ range for strong $q$ variations with respect to the family of spherical means $A_{t} f(x)=f * \sigma_{t}(x)$ defined in (17). To obtain the first part of Theorem 1.4 it suffices by Theorem 1.1, (16) and Lemma 2.1 to bound the short $q$-variation operator $S_{q}(\mathcal{A} f)$ and prove

$$
\left\|S_{q}(\mathcal{A} f)\right\|_{p} \leq C_{p, q}\|f\|_{p}
$$

for $d /(d-1)<p<2 d$, with $q=2$ if $d \geq 3$ and $q>2$ if $d=2$. Interpolation with the trivial $L^{\infty}$ estimate for the corresponding maximal operator shows that for $p \geq 2 d$, $L^{p}$ estimates hold for strong $q$-variations whenever $q>p / d$. To bound $S_{q}(\mathcal{A} f)$ we employ again the Littlewood-Paley decomposition $\mathcal{A}^{k}=\left\{A_{t}^{k}\right\}$ as it appears in the proof of Lemma 6.1; thus for $k>0$,

$$
\widehat{A_{t}^{k}} f(\xi)=\widehat{\sigma}(t \xi) \psi\left(2^{-k} t \xi\right) \widehat{f}(\xi)
$$

with the usual modification for $k=0$. We estimate $S_{q}(\mathcal{A} f) \leq \sum_{k=0}^{\infty} S_{q}\left(\mathcal{A}^{k} f\right)$ as before. It suffices to consider the case $k>0$ as the case $k=0$ is certainly covered by Lemma 6.1. We now formulate a lemma which is used together with the 1-dimensional embedding (39) in the $t$ variable (for $\rho=2$ ) to estimate $S_{2}\left(\mathcal{A}^{k} f\right)$. The lemma is based on results for square-functions associated to Bochner-Riesz operators in [11, [15], 40]; see also [30], 34]. 
Lemma 8.1. Suppose that $d \geq 2$ and $p \geq 2(d+1) /(d-1)$, or $d=2, p \geq 4$. Then

$$
\begin{array}{r}
\left\|\left(\int_{1}^{2}\left|A_{t}^{k} f\right|^{2} d t\right)^{1 / 2}\right\|_{L^{p}\left(\mathbb{R}^{d}\right)}+2^{-k}\left\|\left(\int_{1}^{2}\left|(d / d t) A_{t}^{k} f\right|^{2} d t\right)^{1 / 2}\right\|_{L^{p}\left(\mathbb{R}^{d}\right)} \\
\leq C_{p} 2^{k(d[1 / 2-1 / p]-1 / 2+\epsilon-(d-1) / 2)}\|f\|_{p} .
\end{array}
$$

Define the operators $W_{k, t}^{ \pm}$by

$$
\widehat{W_{k, t}^{ \pm} f}=a_{k}(|\xi|) e^{ \pm i|\xi| t} \widehat{f}(\xi),
$$

where $a_{k}$ is a standard symbol of order 0 supported in $\left(2^{k-2}, 2^{k+2}\right)$. Note that $A_{t}^{k}$ is a linear combination of operators of the form $2^{-k(d-1) / 2} W_{k, t}^{ \pm}$with suitable choices of the symbols (and $(d / d t) A_{t}^{k}$ is a linear combination of operators of the form $2^{-k(d-3) / 2} W_{k, t}^{ \pm}$.

Let $I$ be a compact interval. We then have the inequality

$$
\left\|\left(\int_{I}\left|W_{k, t}^{ \pm} f(x)\right|^{2} d t\right)^{1 / 2}\right\|_{p} \lesssim C_{\varepsilon} 2^{k(d(1 / 2-1 / p)-1 / 2+\epsilon)}\|f\|_{p}
$$

for $4<p<\infty$ and $d=2$ and for $2(d+1) /(d-1)<p<\infty, d \geq 3$. This is well known and closely related to the estimate

$$
\left\|\left(\int_{s \approx 1}\left|\mathcal{F}^{-1}\left[\zeta\left(2^{k}\left(1-\frac{|\cdot|}{s}\right)\right) \widehat{f}\right]\right|^{2} d s\right)^{1 / 2}\right\|_{p} \leq C_{\varepsilon}(\zeta) 2^{k(d(1 / 2-1 / p)-1+\epsilon)}\|f\|_{p}
$$

for $\zeta \in C_{0}^{\infty}$ supported in, say, $(-1 / 4,1 / 4)$. The latter inequality has been shown by Carbery [11] when $d=2, p=4$ and by Christ [15] and one of the authors [40] if $p \geq 2(d+1) /(d-1)$. We note that the assumption of $\zeta \in C_{0}^{\infty}(-1 / 4,1 / 4)$ could be relaxed by assuming that $\zeta \in \mathcal{S}(\mathbb{R})$ by decomposing $\zeta$ into dyadic pieces. For the dependence on $\zeta$ we have

$$
C_{\varepsilon}(\zeta) \leq c_{\varepsilon}\|\zeta\|_{*},
$$

where the norm can be chosen to be $\|\zeta\|_{*}:=\sup _{s} \sum_{j=0}^{M}\left|\zeta^{(j)}(s)\right|(1+|s|)^{M}$ for suitably large $M=M(d)$.

To relate the inequalities (53) and (54) we follow in spirit Kaneko-Sunouchi [30] (see also 34 for related arguments). We choose $\chi \in \mathcal{S}(\mathbb{R})$ so that the Fourier transform of $\chi$, henceforth denoted by $\chi_{0}$, is compactly supported in $[-1,1]$ and $\chi(s)>0$ on $[1,2]$. Then

$$
\begin{array}{r}
\left(\int_{1}^{2}\left|W_{k, t}^{ \pm} f(x)\right|^{2} d t\right)^{1 / 2} \lesssim\left(\int_{-\infty}^{\infty}\left|\chi(t) W_{k, t}^{ \pm} f(x)\right|^{2} d t\right)^{1 / 2} \\
=c\left(\int_{-\infty}^{\infty}\left|\int \chi(t) W_{k, t}^{ \pm} f(x) e^{-i \tau t} d t\right|^{2} d \tau\right)^{1 / 2}
\end{array}
$$

and

$$
\int \chi(t) W_{k, t}^{ \pm} f(x) e^{-i \tau t} d t=\mathcal{F}^{-1}\left[m_{\tau}(|\cdot|) \widehat{f}\right]
$$

where

$$
m_{\tau}(s)=\chi_{0}(\tau \mp s) a_{k}(s) .
$$


By scaling, the desired estimate follows from

$$
\begin{aligned}
\|\left(\int_{|\tau| \approx 2^{k}}\left|\mathcal{F}^{-1}\left[\chi_{0}\left(\tau-2^{k}|\cdot|\right) a_{k}\left(2^{k}|\cdot|\right) \widehat{f}\right]\right|^{2} d \tau\right)^{1 / 2} & \|_{p} \\
& \leq C_{\varepsilon} 2^{k(d(1 / 2-1 / p)-1 / 2+\epsilon)}\|f\|_{p},
\end{aligned}
$$

where $\eta=a_{k}\left(2^{k} \cdot\right)$ satisfies uniform estimates in $k$. After a change of variable in $\tau$ we have reduced matters to the inequality

$$
\left\|\left(\int_{1 \leq|\sigma| \leq 2}\left|\mathcal{F}^{-1}\left[\chi_{0}\left(2^{k}(\sigma-|\cdot|)\right) \widehat{f}\right]\right|^{2} d \sigma\right)^{1 / 2}\right\|_{p} \leq C_{\varepsilon} 2^{k(d(1 / 2-1 / p)-1+\epsilon)}\|f\|_{p} .
$$

The estimates needed to prove (55) are exactly the same as needed for (54). However one can in fact deduce (55) from (54). Let $\eta \in C_{0}^{\infty}(\mathbb{R})$ so that $\eta(\sigma)=1$ on $(1 / 2,4)$ and $\eta$ is supported on $(1 / 4,5)$. Let

$$
u(s, \sigma)=\eta(\sigma) \chi_{0}(\sigma s)
$$

so that $u$ is smooth and compactly supported in both variables. Let $\lambda \mapsto w_{\lambda}(s)$ be the partial Fourier transform of $u$ with respect to $\sigma$ and write

$$
u(s, \sigma)=(2 \pi)^{-1} \int w_{\lambda}(s) e^{i \lambda \sigma} d \lambda .
$$

Then $w$ is compactly supported in the $s$ variable and all derivatives decay fast with respect to $\lambda$; i.e., $\left\|w_{\lambda}\right\|_{*} \leq C_{N}(1+|\lambda|)^{-N}$. Now

$$
\begin{aligned}
\eta(\sigma) \mathcal{F}^{-1}\left[\eta(\sigma) \chi_{0}\left(2^{k}(\sigma-|\cdot|)\right) \widehat{f}\right] & =\mathcal{F}^{-1}\left[u\left(2^{k}\left(1-\frac{|\cdot|}{\sigma}\right), \sigma\right) \widehat{f}\right] \\
& =(2 \pi)^{-1} \int e^{i \lambda \sigma} \mathcal{F}^{-1}\left[w_{\lambda}\left(2^{k}\left(1-\frac{|\cdot|}{\sigma}\right)\right) \widehat{f}\right] d \lambda .
\end{aligned}
$$

Thus by (54),

$$
\begin{aligned}
& \left\|\left(\int_{1 \leq|\sigma| \leq 2}\left|\mathcal{F}^{-1}\left[\chi_{0}\left(2^{k}(\sigma-|\cdot|)\right) \widehat{f}\right]\right|^{2} d \sigma\right)^{1 / 2}\right\|_{p} \\
& \lesssim \int_{\lambda}\left\|\left(\int_{1 \leq|\sigma| \leq 2}\left|\mathcal{F}^{-1}\left[w_{\lambda}\left(2^{k}\left(1-\frac{|\cdot|}{\sigma}\right)\right) \widehat{f}\right]\right|^{2} d \sigma\right)^{1 / 2}\right\|_{p} \\
& \leq C_{\varepsilon}^{\prime} \int\left\|w_{\lambda}\right\|_{*} d \lambda 2^{k(d(1 / 2-1 / p)-1+\epsilon)}\|f\|_{p}
\end{aligned}
$$

and the integral in $\lambda$ converges.

To bound $\left\|S_{2}\left(\mathcal{A}_{k} f\right)\right\|_{p}$ we need the following global version of Lemma 8.1 ,

Lemma 8.2. Suppose that $d \geq 2$ and $p \geq 2(d+1) /(d-1)$, or $d=2, p \geq 4$. Then for $k>0$,

$$
\begin{aligned}
\left\|\left(\int_{0}^{\infty}\left|A_{t}^{k} f\right|^{2} \frac{d t}{t}\right)^{1 / 2}\right\|_{L^{p}\left(\mathbb{R}^{d}\right)}+2^{-k} \| & \left(\int_{0}^{\infty}\left|t(d / d t) A_{t}^{k} f\right|^{2} \frac{d t}{t}\right)^{1 / 2} \|_{L^{p}\left(\mathbb{R}^{d}\right)} \\
& \leq C_{p, \epsilon} 2^{k(d(1 / 2-1 / p)-1 / 2+\epsilon-(d-1) / 2)}\|f\|_{p} .
\end{aligned}
$$

To prove this we could use weighted norm inequalities as in [11, [15], or use $L^{p}$-Calderón-Zygmund theory as in [40]. We simply quote a general version of the latter, namely a vector-valued version of a result from [41] ( $c f$. also [13]). Here 
one is given a family of Fourier multipliers $m_{s}, 1 \leq s \leq 2$, compactly supported in $\{\xi: 1 / 2 \leq|\xi| \leq 2\}$, and it is assumed that

$$
\left\|\left(\int_{1}^{2}\left|\mathcal{F}^{-1}\left[m_{s}\left(2^{j} \cdot\right) \widehat{f}\right]\right|^{2} d s\right)^{1 / 2}\right\|_{p} \leq A\|f\|_{p}
$$

for some $p \in(1, \infty)$, uniformly in $j \in \mathbb{Z}$. One also assumes that

$$
\left|\partial_{\xi}^{\alpha} m_{s}(\xi)\right| \leq B,|\alpha| \leq d+1,
$$

for some possibly very large constant $B$. The conclusion is that

$$
\left\|\left(\sum_{j} \int_{1}^{2}\left|\mathcal{F}^{-1}\left[m_{s}\left(2^{j} \cdot\right) \widehat{f}\right]\right|^{2} d s\right)^{1 / 2}\right\|_{p} \leq C_{p} A(\log (2+B / A))^{|1 / p-1 / 2|}\|f\|_{p} .
$$

We use this with $B=O\left(2^{k(d+1)}\right)$ and with $A$ being the constant in Lemma 8.1. Thus we only get an irrelevant power of $k$ when passing from Lemma 8.1 to Lemma 8.2. From (39) we obtain that if $d \geq 3$, then

$$
\left\|S_{2}\left(\mathcal{A}^{k} f\right)\right\|_{p} \leq C 2^{-k a(p)}\|f\|_{p}
$$

with $a(p)>0$ when $2(d+1) /(d-1) \leq p<2 d$, which covers the range $d \leq p<2 d$ that does not fall under the scope of Lemma 6.1. Thus we may sum in $k$ to obtain the claimed result for $S_{2}(\mathcal{A} f)$.

In two dimensions the estimates for $S_{2}\left(\mathcal{A}^{k} f\right)$ do not sum and we obtain from Lemma 6.1 and Lemma 8.1 only that

$$
\left\|S_{2}\left(\mathcal{A}^{k} f\right)\right\|_{p} \leq C_{\varepsilon} 2^{k \varepsilon}\|f\|_{p}, \quad 2 \leq p \leq 4, \quad d=2 .
$$

We also need to use a result from 33 . on local smoothing, which says that for $p>2$,

$$
\left\|\left(\int_{I}\left|A_{t}^{k} f\right|^{p}+\left|2^{-k}(d / d t) A_{t}^{k} f\right|^{p} d t\right)^{1 / p}\right\|_{L^{p}\left(\mathbb{R}^{2}\right)} \leq C_{p} 2^{-k(1 / p+\delta(p))}\|f\|_{p}
$$

with some $\delta(p)>0$ (the precise value is irrelevant for our purpose). Since $p>2$ the following global version follows immediately from a straightforward application of the Littlewood-Paley theory.

Lemma 8.3. Suppose that $d=2,2<p<\infty$ and $\varepsilon>0$. Then for $k>0$,

$$
\begin{aligned}
& \left\|\left(\int_{0}^{\infty}\left|A_{t}^{k} f\right|^{p} \frac{d t}{t}\right)^{1 / p}\right\|_{L^{p}\left(\mathbb{R}^{d}\right)} \leq C_{p, \epsilon} 2^{-k(1 / p+\delta(p)-\varepsilon)}\|f\|_{p}, \\
& \left\|\left(\int_{0}^{\infty}\left|t \frac{d}{d t} A_{t}^{k} f\right|^{p} \frac{d t}{t}\right)^{1 / p}\right\|_{L^{p}\left(\mathbb{R}^{d}\right)} \leq C_{p, \epsilon} 2^{k} 2^{-k(1 / p+\delta(p)-\varepsilon)}\|f\|_{p} .
\end{aligned}
$$

This lemma yields $\left\|S_{p}\left(\mathcal{A}^{k} f\right)\right\|_{p} \leq C 2^{-k b(p)}\|f\|_{p}, 2<p<\infty$ and by interpolation with the $L^{p}$-estimate for $S_{2}\left(A^{k} f\right)$ we also get

$$
\left\|S_{q}\left(\mathcal{A}^{k} f\right)\right\|_{p} \leq C 2^{-k b(p, q)}\|f\|_{p}
$$

with $b(p, q)>0$ when $2<p \leq 4, q>2$. This implies the claimed result in two dimensions.

Sharpness. We now turn to the second part of the statement in Theorem 1.4 and show that the a priori inequality

$$
\left\|V_{q}(\mathcal{A} f)\right\|_{p} \leq C_{p}\|f\|_{p}
$$


for $p>2 d$ implies that $q \geq p / d$. This shows that Theorem 1.4 is essentially sharp. We shall test $V_{q}(\mathcal{A} f)(x)$ on the function $f_{\lambda}$ given by

$$
\widehat{f_{\lambda}}(\xi)=\chi\left(\lambda^{-1}|\xi|\right) e^{i|\xi|^{2} /(2 \lambda)} ;
$$

here $\chi \in C_{0}^{\infty}$ is nonnegative, supported in $[1 / 2,3 / 2]$ and $\chi \equiv 1$ on $[3 / 4,5 / 4]$. When deriving a lower bound for $V_{q}(\mathcal{A} f)(x)$ we restrict $x$ to a ball of radius $c_{0} \lambda^{-1}$, centered at the origin.

After a change of variable we may write

$$
f_{\lambda}(y)=\left(\frac{\lambda}{2 \pi}\right)^{d} \int \chi(|\eta|) e^{i \lambda\left[|\eta|^{2} / 2+\langle x, \eta\rangle\right]} d \eta .
$$

Clearly $f_{\lambda}$ decays rapidly when $|y| \geq 2$ and by the method of stationary phase we have $\left|f_{\lambda}(y)\right| \leq C \lambda^{d / 2}$, so that $\left\|f_{\lambda}\right\|_{p}=O\left(\lambda^{d / 2}\right)$ for all $p$. Now recall that

$$
\widehat{A_{t} f}(\xi)=c_{d} \frac{J_{\alpha}(t|\xi|)}{(t|\xi|)^{\alpha}} \widehat{f}(\xi), \quad \alpha=(d-2) / 2
$$

so that

$$
\begin{aligned}
c_{d}^{-1} A_{t} f_{\lambda}(x) & =\frac{1}{(2 \pi)^{d}} \int \chi\left(\lambda^{-1}|\xi|\right) e^{i|\xi|^{2} /(2 \lambda)} \frac{J_{\alpha}(t|\xi|)}{(t|\xi|)^{\alpha}} e^{i\langle x, \xi\rangle} d \xi \\
& =\left(\frac{\lambda}{2 \pi}\right)^{d} \int \chi(r) r^{d-1} e^{i \lambda r^{2} / 2} \frac{J_{\alpha}(\lambda t r)}{(\lambda t r)^{\alpha}} \vartheta(\lambda r|x|) d r
\end{aligned}
$$

where $\vartheta(s)=\int_{\theta \in S^{d-1}} e^{i s\left\langle\frac{x}{|x|}, \theta\right\rangle} d \sigma(\theta)$ is independent of $x$. Furthermore $\vartheta(s)=$ $\varpi_{d}+O(s)$, where $\varpi_{d}$ is the total surface measure of the unit sphere $S^{d-1}$ and the derivatives of $\vartheta$ stay bounded if $|s| \leq 10$.

For $R \geq 1$,

$$
J_{\alpha}(R)=\sqrt{\frac{2}{\pi R}} \cos \left(R-\frac{\pi \alpha}{2}-\frac{\pi}{4}\right)+O\left(R^{-3 / 2}\right) .
$$

Thus we have

$$
c_{d}^{-1} A_{t} f(x)=[I+I I+I I I](x, t)
$$

where

$$
\begin{aligned}
I(x, t) & =\left(\frac{\lambda}{2 \pi}\right)^{d} e^{i\left(\frac{\pi \alpha}{2}+\frac{\pi}{4}\right)} \int \chi(r) r^{d-1}(2 \pi \lambda r t)^{-1 / 2-\alpha} e^{i \lambda\left(r^{2} / 2-r t\right)} \vartheta(\lambda|x| r) d r, \\
I I(x, t) & =\left(\frac{\lambda}{2 \pi}\right)^{d} e^{-i\left(\frac{\pi \alpha}{2}+\frac{\pi}{4}\right)} \int \chi(r) r^{d-1}(2 \pi \lambda r t)^{-\alpha-1 / 2} e^{i \lambda\left(r^{2} / 2+r t\right)} \vartheta(\lambda|x| r) d r, \\
I I I(x, t) & =O\left(\lambda^{d-\alpha-3 / 2}\right), \quad t \in[1 / 2,2] .
\end{aligned}
$$

Notice that the phase for $I I(x, t)$ does not have any critical points if $t>0$, and therefore $I I(x, t)$ can be subsumed under the error term. The asymptotics of the integral for $I(x, t)$ are obtained by the method of stationary phase; the phase has a nondegenerate critical point at $r=t$.

We get for $t \approx 1,|c| \leq c_{0} \lambda^{-1}$,

$$
I(x, t)=\tilde{c}_{d} \lambda^{d-\alpha-1} \chi(t) t^{d-2-2 \alpha} e^{-i \lambda t^{2} / 2} \vartheta(\lambda|x| r)+O\left(\lambda^{d-\alpha-3 / 2}\right) .
$$

For $1 \leq n \leq \lambda / 100$, choose

$$
t_{n}=\sqrt{2+\frac{2 n \pi}{\lambda}}
$$


then $\left|e^{-i \lambda t_{n}^{2} / 2}-e^{-i \lambda t_{n+1}^{2} / 2}\right|=2$ and $\left|t_{n}-t_{n+1}\right|=O\left(\lambda^{-1}\right)$. Therefore

$$
\left(\sum_{1 \leq n<\frac{\lambda}{100}}\left|I\left(x, t_{n}\right)-I\left(x, t_{n+1}\right)\right|^{q}\right)^{1 / q} \geq \lambda^{d-\alpha-1+1 / q}\left(c-C \lambda^{-1}\right) .
$$

The corresponding expressions for $I$ replaced by $I I$ and $I I I$ have the upper bound $O\left(\lambda^{d-\alpha-3 / 2+1 / q}\right)$.

Now recall that $\alpha=(d-2) / 2$. Combining the terms above yields for $|x| \leq c_{0} \lambda^{-1}$, $c_{0}$ small, the estimate

$$
\left|V_{q}\left(\mathcal{A} f_{\lambda}\right)(x)\right| \geq c \lambda^{d / 2+1 / q},
$$

and consequently $\left\|V_{q}\left(\mathcal{A} f_{\lambda}\right)\right\|_{p} \geq c^{\prime} \lambda^{d / 2+1 / q-d / p}$. Since $\left\|f_{\lambda}\right\|_{p} \leq C \lambda^{d / 2}$ this yields the restriction $q \geq p / d$.

Proof of the restricted weak type endpoint inequalities. As before, since the dyadic jump inequalities hold for all $1<p<\infty$, both the claimed jump and variational restricted weak type inequalities for $p=d /(d-1), d \geq 3$ follow from a shortvariation result

$$
\left\|S_{2}(\mathcal{A} f)\right\|_{L^{d /(d-1), \infty}} \leq C\|f\|_{L^{d /(d-1), 1}} .
$$

This in turn follows by a generalization of an argument by Bourgain 3 , (see the appendix of [14]) from the standard $L^{2}$ bound

$$
\left\|S_{2}\left(\mathcal{A}^{k} f\right)\right\|_{2} \leq C 2^{-k(d-2) / 2}\|f\|_{2}
$$

and the Hardy-space bound

$$
\left\|S_{2}\left(\mathcal{A}^{k} f\right)\right\|_{L^{1}} \leq C 2^{k}\|f\|_{H^{1}}
$$

note that (57) is an improvement by a logarithmic factor of the result following from (46). To show (57) we only need to check the estimate on atoms; we use arguments from [45] (see also [17, [43]). By dilation invariance it suffices to check that

$$
\left\|S_{2}\left(\mathcal{A}^{k} f_{0}\right)\right\|_{1}=O\left(2^{k}\right)
$$

for an atom $f_{0}$ associated to the unit ball $B_{0}$ centered at the origin; i.e., $f_{0}$ is supported on $B_{0},\left\|f_{0}\right\|_{\infty} \leq 1$ and $\int f_{0}=0$. It suffices to show

$$
\left\|\left(\sum_{j}\left[\int_{1}^{2}\left|\frac{d}{d s} A_{2^{j} s}^{k} f_{0}\right| d s\right]^{2}\right)^{1 / 2}\right\|_{1} \leq C 2^{k},
$$

which in turn follows from

$$
\left\|\left(\sum_{j}\left|\frac{d}{d s} A_{2^{j} s}^{k} f_{0}\right|^{2}\right)^{1 / 2}\right\|_{1} \leq C 2^{k},
$$

uniformly in $s$. For each $s \in[1,2], k>0$, let $\mathcal{U}_{k, s}$ be the set of all $x$ for which either $|x| \leq 2$ or ||$x\left|-2^{j} s\right| \leq 2$ for some $0<j \leq k$. Then the measure of $\mathcal{U}_{k, s}$ is $O\left(2^{k(d-1)}\right)$. Now the inequality (58) follows from

$$
\begin{aligned}
& \left\|\left(\sum_{j \leq k+10}\left|\frac{d}{d s} A_{2^{j} s}^{k} f_{0}\right|^{2}\right)^{1 / 2}\right\|_{L^{1}\left(\mathcal{U}_{k, s}\right)} \leq C 2^{k}, \\
& \left\|\frac{d}{d s} A_{2^{j} s}^{k} f_{0}\right\|_{L^{1}\left(\mathbb{R}^{d} \backslash \mathcal{U}_{k, s}\right)} \leq C 2^{j}, \quad j \leq k, \\
& \left\|\frac{d}{d s} A_{2^{j_{s}}}^{k} f_{0}\right\|_{L^{1}\left(\mathbb{R}^{d}\right)} \leq C 2^{2 k-j}, \quad j>k .
\end{aligned}
$$


To see (59) we apply the Cauchy-Schwarz inequality on the exceptional set $\mathcal{U}_{k, s}$ and from the standard $L^{2}$ estimates we get

$$
\begin{aligned}
& \left\|\left(\sum_{j \leq k+10}\left|\frac{d}{d s} A_{2^{j} s}^{k} f_{0}\right|^{2}\right)^{1 / 2}\right\|_{L^{1}\left(\mathcal{U}_{k, s}\right)} \\
& \leq C 2^{k(d-1) / 2}\left\|\left(\sum_{j \leq k+10}\left|\frac{d}{d s} A_{2^{j} s}^{k} f_{0}\right|^{2}\right)^{1 / 2}\right\|_{2} \\
& \leq C 2^{k(d-1) / 2} 2^{-k(d-3) / 2}\left\|f_{0}\right\|_{2} \leq C^{\prime} 2^{k} .
\end{aligned}
$$

If $\mathcal{K}_{k, j, s}$ denotes the convolution kernel of $(d / d s) A_{2^{j} s}^{k}$, then

$$
\left|\mathcal{K}_{k, j, s}(x)\right|+2^{j-k}\left|\nabla \mathcal{K}_{k, j, s}(x)\right| \leq C_{N} 2^{2 k}\left(1+2^{k}\left|\frac{|x|}{2^{j} s}-1\right|\right)^{-N}
$$

and using this estimate for $\mathcal{K}_{k, j, s}$ away from the exceptional set implies (60). Using the gradient estimate and the cancellation of $f_{0}$ one obtains (61). For more details, see [45], 43]; we remark that the methods of these papers yield in fact improved jump and variational inequalities for families of spherical means with the dilation parameter restricted to subsets of $\mathbb{R}^{+}$.

\section{REFERENCES}

[1] M. Akcoglu, R.L. Jones and P. Schwartz, Variation in probability, ergodic theory and analysis, Illinois J. Math., 42 (1998), 154-177. MR.1492045 (99a:60048)

[2] J. Bergh and J. Peetre, On the spaces $V_{p}, 0<p<\infty$, Bollettino U.M.I., 10 (1974), 632-648. MR0380389 (52:1289)

[3] J. Bourgain, Estimations de certaines fonctions maximales, C.R. Acad. Sci. Paris, 301 (1985), 499-502. MR812567 (87b:42023)

[4] Averages in the plane over convex curves and maximal operators, J. Analyse Math., 47 (1986), 69-85. MR874045 (88f:42036)

[5] - Pointwise ergodic theorems for arithmetic sets, Inst. Hautes Études Sci. Publ. Math. 69 (1989), 5-45. MR:1019960 (90k:28030)

[6] D.L. Burkholder, B. Davis and R. Gundy, Integral inequalities for convex functions of operators on martingales, Proc. Sixth Berkeley Symp. Math. Statist. Prob., 2 (1972), 223-240. MR0400380 (53:4214)

[7] A.P. Calderón, Ergodic theory and translation invariant operators, Proc. Nat. Acad. Sci. USA, 59 (1968), 349-353. MR0227354 (37:2939)

[8] A.P. Calderón and A. Zygmund, On singular integrals, Amer. J. Math. 78 (1956), 289-309. MR0084633 (18:894a)

[9] J. Campbell, R.L. Jones, K. Reinhold and M. Wierdl, Oscillation and variation for the Hilbert transform, Duke Math. J., 105 (2000), 59-83. MR1788042 (2001h:42021)

[10] _ Oscillation and variation for singular integrals in higher dimensions, Trans. Amer. Math. Soc., 35 (2003), 2115-2137. MR1953540 (2003j:44007)

[11] A. Carbery, The boundedness of the maximal Bochner-Riesz operator on $L^{4}\left(\mathbb{R}^{2}\right)$, Duke Math. J., 50 (1983), 409-416. MR705033 (84m:42025)

[12] _ Radial Fourier multipliers and associated maximal functions, Recent progress in Fourier analysis, ed. by I. Peral and J.L. Rubio de Francia, North Holland, 1985. MR848141 (87i:42029)

[13] Variants of the Calderón-Zygmund theory for $L^{p}$-spaces, Rev. Mat. Iberoamericana 2 (1986), no. 4, 381-396. MR913694 (89f:42011)

[14] A. Carbery, A. Seeger, S. Wainger and J. Wright, Classes of singular integral operators along variable lines, J. Geom. Anal. 9 (1999), 583-605. MR1757580(2001g:42026)

[15] M. Christ, On the almost everywhere convergence of Bochner-Riesz means in higher dimensions, Proc. Amer. Math. Soc., 95 (1985), 16-20. MR796439 (87c:42020)

[16] _ $\quad$ A T $(b)$ theorem with remarks on analytic capacity and the Cauchy integral, Colloq. Math., 60/61 (1990), 601-628. MR.1096400 (92k:42020) 
[17] Weak type $(1,1)$ bounds for rough operators, Ann. of Math., 128 (1988), 19-42. MR951506 (89m:42013)

[18] M. Christ and J.L. Rubio de Francia, Weak type $(1,1)$ bounds for rough operators. II. Invent. Math. 93 (1988), no. 1, 225-237. MR943929 (90d:42021)

[19] H. Dappa and W. Trebels, On maximal functions generated by Fourier multipliers, Ark. Mat., 23 (1985), 241-259. MR827345 (87h:42025)

[20] G. David, Morceaux de graphes lipschitziens et intégrales singulières sur une surface, Rev. Mat. Iberoamericana, 4 (1988), 73-114. MR1009120 (90h:42026)

[21] J. Duoandikoetxea and J.-L. Rubio de Francia, Maximal and singular integral operators via Fourier transform estimates, Invent. Math., 84 (1986), 541-561. MR837527 (87f:42046)

[22] J. Garcia-Cuerva and J.L. Rubio de Francia, Weighted norm inequalities and related topics, North Holland, 1985. MR.807149 (87d:42023)

[23] L. Grafakos and N. Kalton, The Marcinkiewicz multiplier condition for bilinear operators, Studia Math. 146 (2001), 151-180. MR1853518(2002f:42007)

[24] S. Hofmann, Weak $(1,1)$ boundedness of singular integrals with nonsmooth kernel, Proc. Amer. Math. Soc. 103 (1988), 260-264. MR938680 (89f:42013)

[25] R.L. Jones, Ergodic averages on spheres, J. Anal. Math. 61 (1993), 29-45. MR1253437 (95g:28031)

[26] R.L. Jones, R. Kaufman, J. Rosenblatt, and M. Wierdl, Oscillation in ergodic theory, Erg. Th. \& Dyn. Sys., 18 (1998), 889-936. MR 1645330 (2000b:28019)

[27] R.L. Jones, J. Rosenblatt, and M. Wierdl, Oscillation inequalities for rectangles, Proc. Amer. Math. Soc., 129 (2000), 1349-1358. MR 1814160 (2001m:42039)

[28] _ Oscillation in ergodic theory: higher dimensional results, Israel Journal of Math., 135 (2003), 1-27. MR.1996394(2004h:37005)

[29] R.L. Jones and G. Wang, Variation inequalities for the Fejér and Poisson kernels, Trans. Amer. Math. Soc., 356 (2004), 4493-4518. MR2067131 (2005c:42005)

[30] M. Kaneko and G. Sunouchi, On the Littlewood-Paley and Marcinkiewicz functions in higher dimensions, Tohoku Math. J. (2) 37 (1985), 343-365. MR799527 (86m:42028)

[31] M. Lacey, Ergodic averages on circles, J. Anal. Math. 67 (1995), 199-206. MR1383493 (97f:28045)

[32] D. Lépingle, La variation d'order p des semi-martingales, Z. Wahrscheinlichkeitstheorie Verw. Gebiete, 36 (1976), 295-316. MR0420837 (54:8849)

[33] G. Mockenhaupt, A. Seeger and C.D. Sogge, Wave front sets, local smoothing and Bourgain's circular maximal function, Ann. of Math., 136 (1992), 207-218. MR.1173929 (93i:42009)

[34] _ Local smoothing of Fourier integral operators and Carleson-Sjölin estimates, J. Amer. Math. Soc., 6 (1993), 65-130. MR.1168960 (93h:58150)

[35] A. Nagel, N. Riviere and S. Wainger, A maximal function associated to the curve $\left(t, t^{2}\right)$, Proc. Nat. Acad. Sci. USA, 73 (1976), 1416-1417. MR0399389(53:3233)

[36] G. Pisier and Q. Xu, The strong p-variation of martingales and orthogonal series, Prob. Theory, 77 (1988), 497-451. MR933985 (89d:60086)

[37] J. Qian, The p-variation of partial sum processes and the empirical process, Ann. of Prob., 26 (1998), 1370-1383. MR1640349 (99i:60052)

[38] G.C. Rota, An "Alternierende Verfahren" for general positive operators, Bull. Amer. Math. Soc., 68 (1962), 95-102. MR0133847 (24:A3671)

[39] J.L. Rubio de Francia, Maximal functions and Fourier transforms, Duke Math. J., 53 (1986), 395-404. MR850542 (87j:42046)

[40] A. Seeger, On quasiradial Fourier multipliers and their maximal functions, J. reine angew. Math., 370 (1986), 61-73. MR852510 (87k:42020)

[41] _ Some inequalities for singular convolution operators in $L^{p}$-spaces, Trans. Amer. Math. Soc. 308 (1988), no. 1, 259-272. MR955772 (89j:42015)

[42] S Singular integral operators with rough convolution kernels, J. Amer. Math. Soc. 9 (1996), 95-105. MR1317232 (96f:42021)

[43] A. Seeger, T. Tao and J. Wright, Endpoint mapping properties for spherical maximal operators, J. Inst. Math. Jussieu 2 (2003), 109-144. MR.1955209 (2003k:42038)

[44] Singular maximal functions and Radon transforms near $L^{1}$, Amer. J. Math., 126 (2004), 607-647. MR2058385 (2005i:44003)

[45] A. Seeger, S. Wainger and J. Wright, Pointwise convergence of spherical means, Math. Proc. Cambridge Phil. Soc. 118 (1995), 115-124. MR.1329463 (96b:42013) 
[46] E.M. Stein, Topics in harmonic analysis related to the Littlewood-Paley theory, Princeton University Press, Princeton, N.J., 1970. MR0252961 (40:6176)

[47] _ Singular Integrals and Differentiability Properties of Functions, Princeton University Press, Princeton, N.J., 1971. MR0290095 (44:7280)

[48] - Maximal functions: Spherical means, Proc. Nat. Acad. Sci. USA, 73 (1976), 21742175. MR0420116(54:8133a)

[49] _ Harmonic Analysis: Real variable methods, orthogonality and oscillatory integrals, Princeton University Press, Princeton, N.J., 1993. MR.1232192 (95c:42002)

[50] E.M. Stein and S. Wainger, Problems in harmonic analysis related to curvature, Bull. Amer. Math. Soc., 84 (1978), 1239-1295. MR508453 (80k:42023)

[51] E.M. Stein and G. Weiss, Introduction to Fourier Analysis on Euclidean Spaces, Princeton University Press, Princeton, N.J., 1971. MR0304972 (46:4102)

[52] T. Tao, The weak-type $(1,1)$ of $L \log L$ homogeneous convolution operator, Indiana Univ. Math. J. 48 (1999), 1547-1584. MR1757083 (2001f:42029)

Department of Mathematics, DePaul University, Chicago, Illinois 60614

Current address: Conserve School, 5400 N. Black Oak Lake Road, Land O'Lakes, Wisconsin 54540

Department of Mathematics, University of Wisconsin, 480 Lincoln Drive, Madison, WISCONSIN 53706

E-mail address: seeger@math.wisc.edu

School of Mathematics, University of Edinburgh, JCMB, King's Buildings, Mayfield Road, Edinburgh EH9 3JZ, Scotland

E-mail address: wright@maths.ed.ac.uk 